\title{
Imaging the Patient with Epilepsy
}

\author{
Charlie Chia-Tsong Hsu, Lars Stenberg, and Timo Krings
}

\section{Learning Objectives}

- To understand typical imaging criteria to identify and differentiate causes of medication refractory epilepsy

- To understand the limitations of imaging for patients with medication refractory epilepsy if no dedicated Epilepsy MR protocol is used.

- To appreciate the importance of additional clinical information when evaluating the patient with medication refractory epilepsy.

C. C.-T. Hsu

Division of Neuroradiology, Department of Medical Imaging, Gold Coast University Hospital, Southport, QLD, Australia

Division of Neuroradiology, Queensland Diagnostic Imaging, Healius Health Care, Varsity Lakes, QLD, Australia

\section{Stenberg}

Section of Neuroradiology, Department of Medical Imaging and Physiology, Skåne University Hospital, Lund, Sweden

Department of Diagnostic Radiology, Lund University, Lund, Sweden

e-mail: Lars.Stenberg@skane.se

T. Krings $(\bowtie)$

Department of Radiology and Surgery, University of Toronto, Toronto, ON, Canada

The David Braley and Nancy Gordon Chair of Interventional Neuroradiology, University of Toronto, Toronto, ON, Canada

Division of Neuroradiology, Toronto Western Hospital, Toronto, ON, Canada

Joint Department of Medical Imaging at the University Health Network, Toronto, ON, Canada

Women's College Hospital, Toronto, ON, Canada

Mount Sinai Hospital, Toronto, ON, Canada

Medical Imaging, Toronto Western Hospital, Toronto, ON, Canada e-mail: timo.krings@uhn.ca

\subsection{Introduction}

Approximately 4\% of the general population will experience a seizure during their lifetime. Imaging findings of "firstever" seizure patients are in most cases normal, and abnormalities are only present in approximately $15 \%$ of patients as seizures can be provoked by fever, sleep deprivation, stroboscopic lights, or drugs. However, an underlying lesion will lower the seizure threshold and thus make a patient more susceptible to experience a seizure. As "first-ever" seizures are a medical emergency the initial imaging modality of choice in these cases is an unenhanced CT head scan to exclude acute medical emergencies that may go along with seizures prior to a more extensive workup depending on clinical history and presentation. Imaging abnormalities encountered in patients experiencing their first-ever seizures include (but are not restricted to) virtually all diseases affecting the brain. As such you may find vascular abnormalities (such as microangiopathy, arteriovenous malformations (AVM), sinus thrombosis, hemorrhage, cavernomas, or stroke), tumors (metastases, primary tumors), infections (encephalitis, meningitis, abscess), sequelae of previous head injury, and toxic or metabolic conditions (e.g., PRES) in these patients.

In contrast to the "first-ever" seizure, patients diagnosed with "epilepsy" have recurrent and unprovoked seizures. Approximately $1 \%$ of the general population will be diagnosed with this condition and as seizures are recurrent and unprovoked, an underlying lesion is far more common as compared to patients with their first-ever seizure. Being "unprovoked," lesions that can irritate the brain (i.e., are "epileptogenic") may be present. On brain imaging, lesions will be seen in nearly $50 \%$ of patients; however, these are non-specific and can encompass a wide variety of underlying conditions that can provoke the recurrent seizure attacks. Imaging findings in patients with seizures include but are not restricted to: vascular conditions such as microangiopathy, previous ischemia, vascular malformations, previous hemor- 
rhage or cavernomas, tumors (metastases, primary tumors), remote infections (encephalitis, abscess), previous head injury, congenital malformations, or toxic metabolic conditions. Imaging of choice in patients with epilepsy is MRI given the larger variety of potential underlying diseases.

The vast majority of patients with epilepsy can be treated satisfactorily with antiepileptic drugs. However, $0.4 \%$ of the general population will have recurrent and unprovoked seizures that do not respond to medication. These patients are potentially treatable with surgery, and surgical intervention is an appropriate consideration for $3 \%$ of people who develop epilepsy [1]. The major focus of this chapter will be on the imaging findings in those patients who are diagnosed with "medication refractory" epilepsy, i.e., patients where the seizure focus is too strong to be controlled by medication which indicates that the underlying lesion has to have a strong epileptogenic potential. In these patients, structural imaging will find abnormalities in up to $85 \%$ of patients. Lesions with a strong epileptogenic potential are either close to epileptogenic structures or consist of abnormal neurons. Lesions that are often involved in medication refractory epilepsy are: mesial temporal lobe sclerosis (MTS) (primary or secondary to a long standing seizure disorder), malformations of cortical development, certain epileptogenic tumors (e.g., dysembryoplastic neuroepithelial tumors (DNET), temporal lobe gliomas gangliogliomas), vascular malformations, trauma, remote infection, and certain phakomatoses. Imaging findings in some of these conditions will be subtle which necessitates both a dedicated imaging protocol (as compared to a standard MR) and an "expert" experience in reading these types of scans. In a landmark study of von Oerzten et al. [2], the sensitivity of "nonexpert" reports of standard MRI reports for focal lesions was $39 \%$, while sensitivity of "expert" reports of standard MRI increased to 50\%. "Expert" reports of epilepsy dedicated MRI protocols further increased the sensitivity in detecting subtle lesions to $91 \%$. Dedicated MRI showed focal lesions in $85 \%$ of patients with "non-lesional" standard MRI. Neuropathological diagnoses were predicted correctly in $22 \%$ of "non-expert" standard MRI reports but by $89 \%$ of dedicated MRI reports. Thus, the combination of dedicated MRI protocols and dedicated radiologists trained in evaluating patients with medication refractory seizures increases significantly the sensitivity of MRI in this subgroup of patients. A multidisciplinary approach that involves close communication between epilepsy neurologists, neuroradiology, EEG, nuclear medicine, neuropsychology, and neurosurgery is an important feature of modern epilepsy management.

The necessity of expert MR reading with a dedicated imaging protocol is further highlighted by the fact that postsurgical seizure freedom is achieved significantly more often when a circumscribed, resectable epileptogenic lesion can be identified on MRI preoperatively compared to patients that are rated non-lesional [3]. As pointed out by Wellmer et al. in
2013 [4, 5], the possible reasons for undetected epileptic lesions in standard outpatient MRI are insufficient clinical information from the referring clinician, routine MR protocols not optimized for the spectrum of epileptogenic lesions, and unfamiliarity with the spectrum of epileptogenic lesions. Wellmer pointed out that "because even the best focus hypothesis and most profound knowledge of epileptogenic lesions do not permit the detection of lesions when they are invisible on the MRI scan, the starting point for any improvement of outpatient MRI diagnostics should be defining an MRI protocol that is adjusted to common epileptogenic lesions."

This indicates that a specific imaging protocol to identify these lesions is necessary. This protocol should take into account that-as small epileptogenic lesions are usually those that provide the best chance for postoperative seizure freedom-slice thickness should be adjusted to detect small lesions and multiple (coronal, axial, sagittal) cut planes are acquired to ensure that physiologic structures or partial volume effects within the folded cortex are not taken for pathology and vice versa. Coronal sequences have to be angulated perpendicular to the hippocampal axis to allow hippocampal volume estimation and caution has to be taken that they are oriented in a plane that ensures direct comparison with the contralateral hemisphere. In our practice, we employ this coronal angulation for both T2FLAIR and T1 IR sequences. High resolution T1-weighted sequences with isotropic voxel sizes allow for multiplanar reformation and further evaluation (including 3D reformats, "pancake" views, surface rendering, and volumetric assessments). T2 gradient echo or susceptibility weighted sequences are highly sensitive to detect blood products or calcifications and should therefore be part of a seizure imaging protocol (Fig. 10.1).

In a recent analysis performed by Wellmer et al. on the prevalence of epileptogenic lesions among 2740 patients the following pathologies were found: mesial temporal lobe sclerosis $(32 \%)$, tumors (including low and high grade tumors as well as malformative tumors and benign epilepsy associated tumors) in approximately $17 \%$ of patients, cortical dysplasias in $11 \%$, glial scars (including posttraumatic, post-ischemic, post-hemorrhagic, postinfectious/abscess, ulegyria, and postsurgical scars) in $11 \%$, vascular diseases (cavernoma AVM, pial angiomatosis) in 5\%, malformations of cortical development including nodular heterotopia, subcortical band heterotopia, polymicrogyria, lissencephaly, pachygyria, agenesis of corpus callosum, craniocephalic malformations, hemiatrophy, lobar dysgenesis, hemimegalencephaly, or hamartomas in $3 \%$, and sequelae of encephalitis in $1 \%$ while in approximately $20 \%$ no lesion could be detected.

Lesion location - presumably related to the different epileptogenic potential in different brain regions - demonstrates preponderance for the temporal lobes $(60 \%)$ followed by the frontal lobe $(20 \%)$, the parietal lobe $(10 \%)$, the periventricu- 

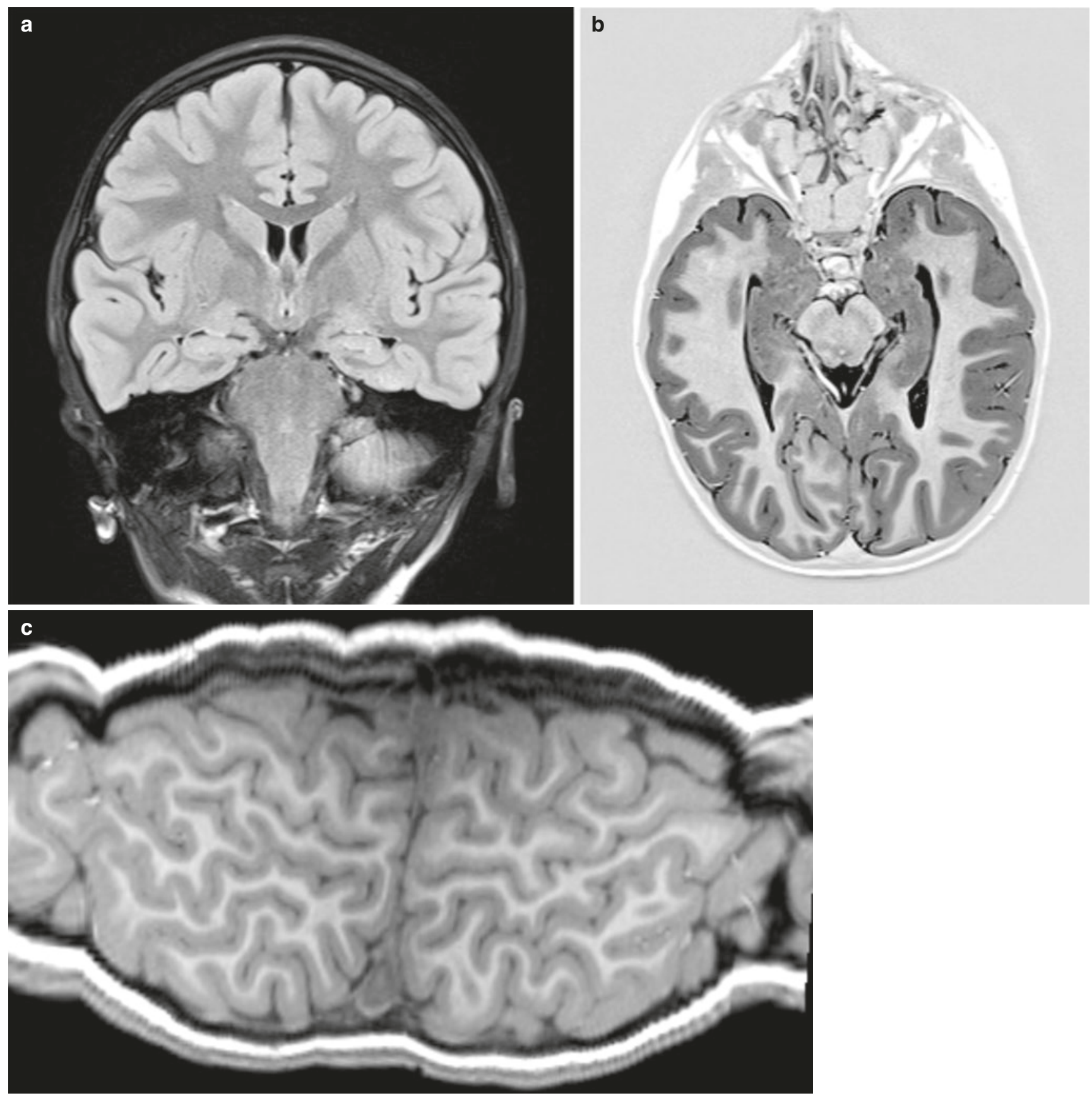

Fig. 10.1 (a-c) Some examples of sequences employed in our institutional dedicated epilepsy protocol: (a) coronal T2 FLAIR, perpendicular to the hippocampal axis; (b) axial T1 inversion recovery (IR) parallel

to the hippocampal axis; (c) T1 3D image set presented as "pancake view" for a better overview of the gyral pattern

higher field strengths (7T) (Fig. 10.2) will probably further increase the detection rate of epileptogenic substrates such as mesial temporal lobe sclerosis (MTS), focal cortical dysplasia (FCD), and polymicrogyria [8-11]. Epilepsy imaging at $1.5 \mathrm{~T}$ is in the opinion of the authors no longer acceptable. There are multiple adjunct imaging techniques: mended that for an epilepsy-specific protocol T2FLAIR, T2/ STIR in two parallel planes, T2 gradient echo/SWI sequences, and an isotropic 3D-T1 are necessary. We strongly recommend these imaging sequences to be done on a 3T scanner given the higher spatial resolution $[6,7]$. The use of even
- Functional MRI (fMRI) can map eloquent cortex and provide information regarding language lateralization [12] (Fig. 10.3), and the use of diffusion tensor imaging (DTI) 


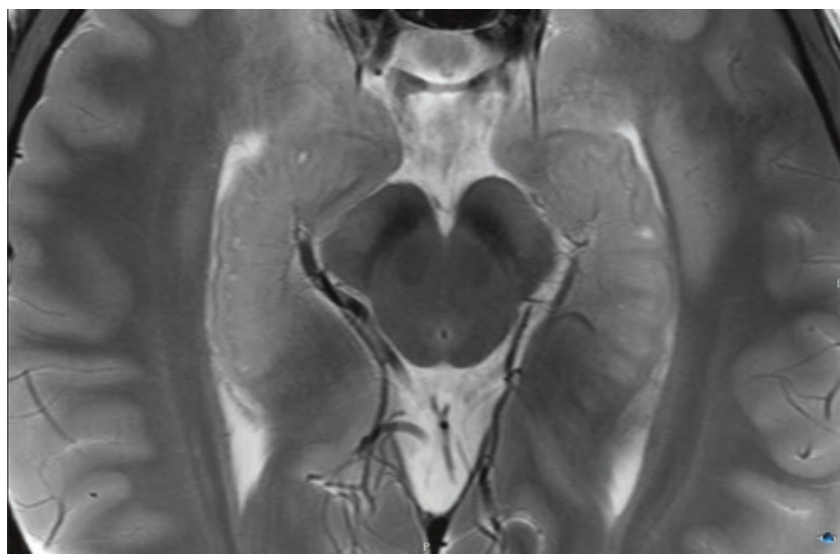

Fig. 10.2 axial T2 at 7T parallel to the hippocampal axis. Excellent in plane resolution provides detailed imaging of hippocampus

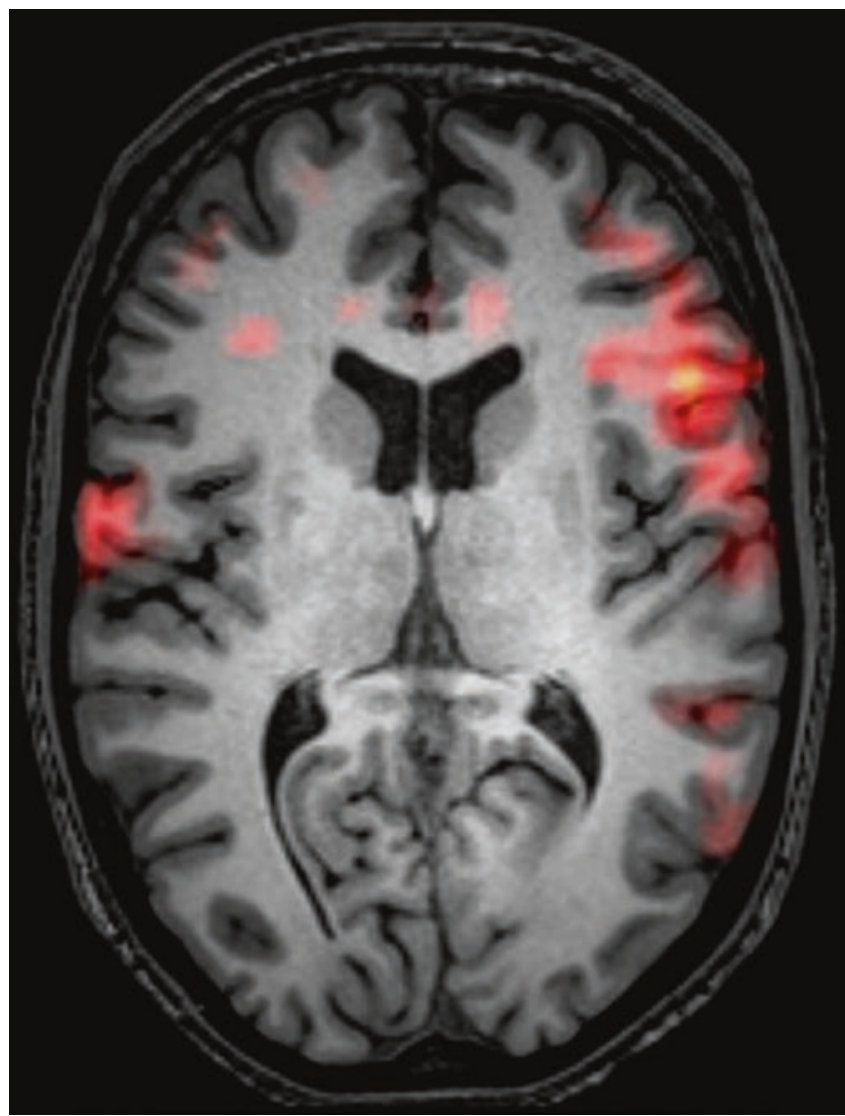

Fig. 10.3 Functional MRI. Red areas indicate activation during a simple word generation task. Activation is seen predominantly in the left hemisphere in the frontal language region; typical language lateralization

and tractography may help to avoid injury to the optic radiation during temporal lobe resection [13].

- Radionuclide imaging can add useful information in selected cases [14]. Subtraction of ictal and interictal SPECT co-registered to MRI (SISCOM) can show a seizure-induced hyperperfusion (Fig. 10.4), whereas ${ }^{18 F}$ FDG-PET may show hypometabolism in the seizure onset zone. This is particularly useful in later- alization of temporal lobe epilepsy in the MR-negative patient.

- Dipole source analysis using either EEG or MEG data can also be added for the localization of seizure activity.

Imaging evaluation should be standardized using a stepwise approach to evaluate the hippocampus and mesial temporal lobe structures, the ventricular outline, the gyral, and the sulcal anatomy. Particular emphasis should be paid upon the T2/FLAIR signal within the cortex and hippocampus, its similarity to other regions of neo- and archicortex, the internal architecture of the hippocampus, the indentations of the pes hippocampi, the fornix and mammillary bodies, and the gray-white matter interface of the neocortex (blurring, gray matter thinning or thickening). Selection of narrow window width better optimizes contrast between the gray and white matter which can assist in lesion detection (Fig. 10.5).

\section{Key Point}

- In selected patients, i.e., those with medication refractory epilepsy, abnormalities can be found in a high percentage if images are performed with a dedicated imaging protocol, and expert read-out. A variety of non-radiological adjunct tests are available that may help in the localization of the seizure focus and preferably these challenging cases are therefore discussed in multidisciplinary conferences.

In the following we will discuss the imaging features of epileptogenic lesions highlighting imaging pearls and pitfalls.

\subsubsection{Mesial Temporal Lobe/Hippocampal Sclerosis}

Patients with mesial temporal sclerosis (MTS) often harbor complex partial seizures with a seizure semiology (given its temporal lobe origin) that is characterized by déjà vu (or jamais vu) sensations, epigastric auras, lip smacking, or other oral automatisms and often have in their past medical history febrile seizures as a child with progressive worsening of seizure frequency and severity over time. Understanding the normal hippocampal internal architecture is critical to make the correct diagnosis. The hippocampus is composed of four distinct cellular layers with stratum oriens as the most superficial layer followed by stratum pyramidale, stratum radiatum, and stratum lacunosum as the inner most layer. Histopathologic correlation with 3T MRI of resected specimen of hippocampi revealed the inner hypointense band on the coronal T2-weighted sequence as the stratum lacunosum (Fig. 10.6). MTS is characterized with extensive gliosis 

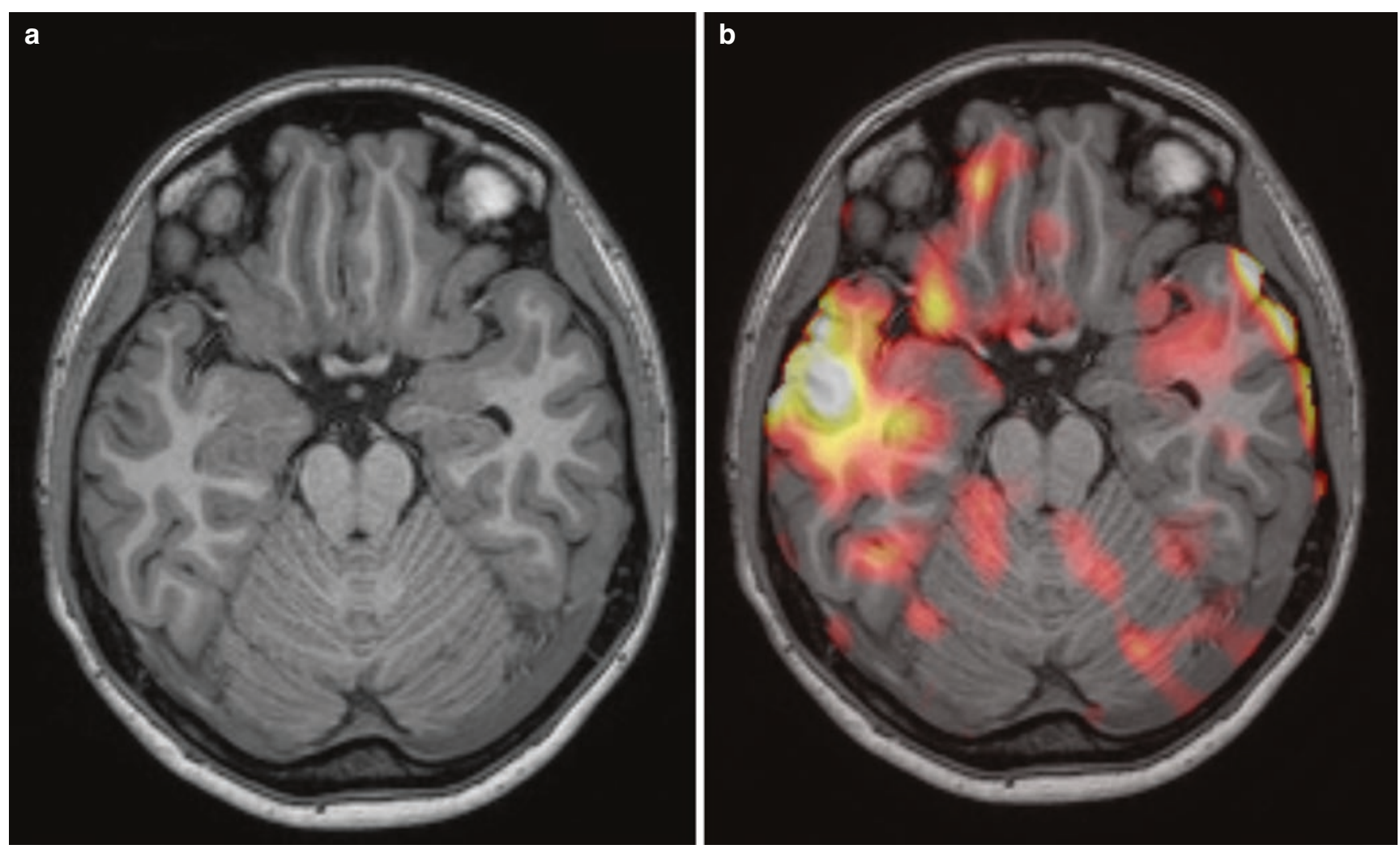

Fig. 10.4 (a, b) SISCOM: (a) axial T1 with small subcortical area in the right temporal lobe with prolonged T1; (b) co-registration of SPECT on MR-images shows ictal hyperperfused area exactly in the same spot as the suspected lesion

throughout the cornu ammonis (CA) regions, but early in the course of the disease there is loss of tissue in the stratum pyramidale most pronounced in CA1 region [15]. Identification of the stratum lacunosum as a landmark provides an imaging reference point for qualitative and quantitative analysis of tissue loss in MTS. Atrophy of the hippocampus and gliosis within the hippocampus will manifest itself as increased signal intensity on T2/FLAIR sequences (Fig. 10.7). The atrophy will lead to loss of the pes hippocampi interdigitations, widening of the temporal horn and atrophy of the white matter of the temporal lobe. As a consequence of Wallerian degeneration, there will be atrophy of the projecting pathways of the hippocampus, i.e., the Papez circuit, with atrophy of the ipsilateral fornix and the mammillary body. In the early stages of MTS the imaging findings are subtle with loss of the internal architecture of the hippocampus with or without associated FLAIR signal changes. Hard windowing of the FLAIR weighted sequences will make identification of the diseased hippocampus easier. In nearly $20 \%$ of patients with MTS, dual pathology is present with a second epileptogenic focus. It is believed that in these cases the other epileptogenic lesion triggered the mesial temporal lobe sclerosis (similar to febrile seizures as a child can trigger or "kindle" a mesial temporal lobe sclerosis). Identification of the second focus is of great importance as failure to do so may result in surgical failure if only a selective amygdalohippocampectomy is performed thus leaving the "primary" focus behind. On the other hand, failure to identify MTS in patients with other lesions may also lead to surgical failure following lesionectomy. Dual pathology may consist also of bilateral mesiotemporal lobe sclerosis as one hemisphere may trigger the other hippocampus to become sclerotic thus constituting bilateral abnormalities. As the internal reference (i.e., the contralateral hippocampus) is similarly affected, comparison of the signal with other regions of archicortex (3-4 layered cortex) can identify whether a mesial temporal lobe sclerosis is present bilaterally. Thus, if the T2/FLAIR signal of the hippocampus is bilateral symmetrical but higher as compared to the cingulum or insula, you have to consider bilateral mesial temporal lobe sclerosis.

\section{Key Point}

- Mesial temporal lobe sclerosis is the most commonly seen cause for medication refractory epilepsy and is characterized by an indistinct gray-white matter differentiation, abnormal high signal on T2/ Flair sequences, and atrophy. In up to $20 \%$ of cases additional epileptogenic pathology is found in patients with mesial temporal lobe sclerosis. 

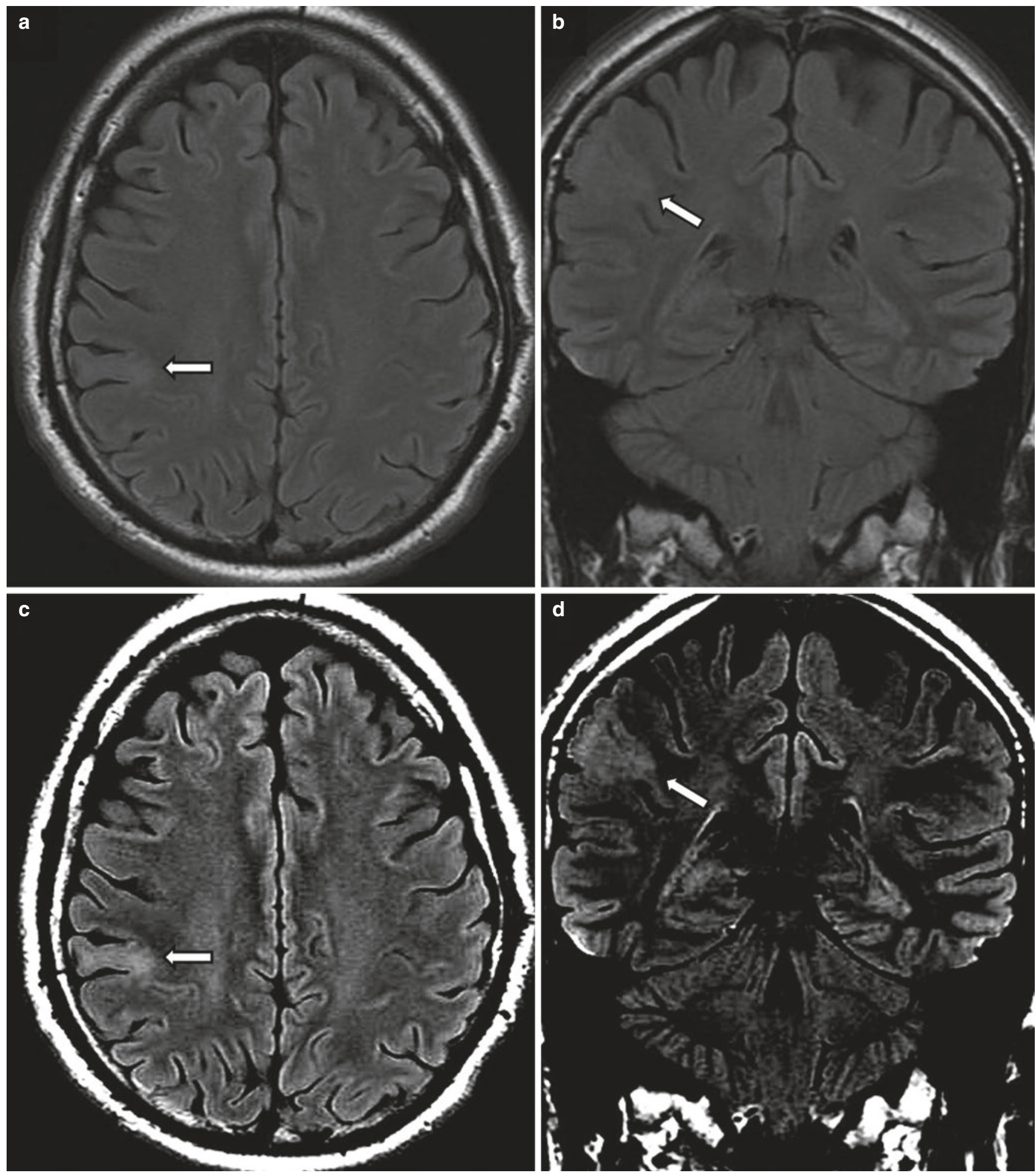

Fig. 10.5 (a, b) Right lateral precentral gyrus type II FCD. (a) axial and (b) coronal FLAIR images at standard window level setting as compared to narrowed window width setting of the same images in $(\mathbf{c}, \mathbf{d})$ which makes the lesion more conspicuous

\subsubsection{Malformations of Cortical Development}

In order to understand the different types of malformations of cortical development it is important to briefly review the embryology of normal brain development: During the seventh week of gestation, neuronal proliferation in the sub- ependymal germinal matrix occurs. After the eighth week these cells migrate outward in multiple waves of radial outward migration aided by radial glial cell guidance in a process coined chemotaxis. The third and last part of the cortical development, the lamination, is the organization of the cells within different cortical layers, a process that is orchestrated 

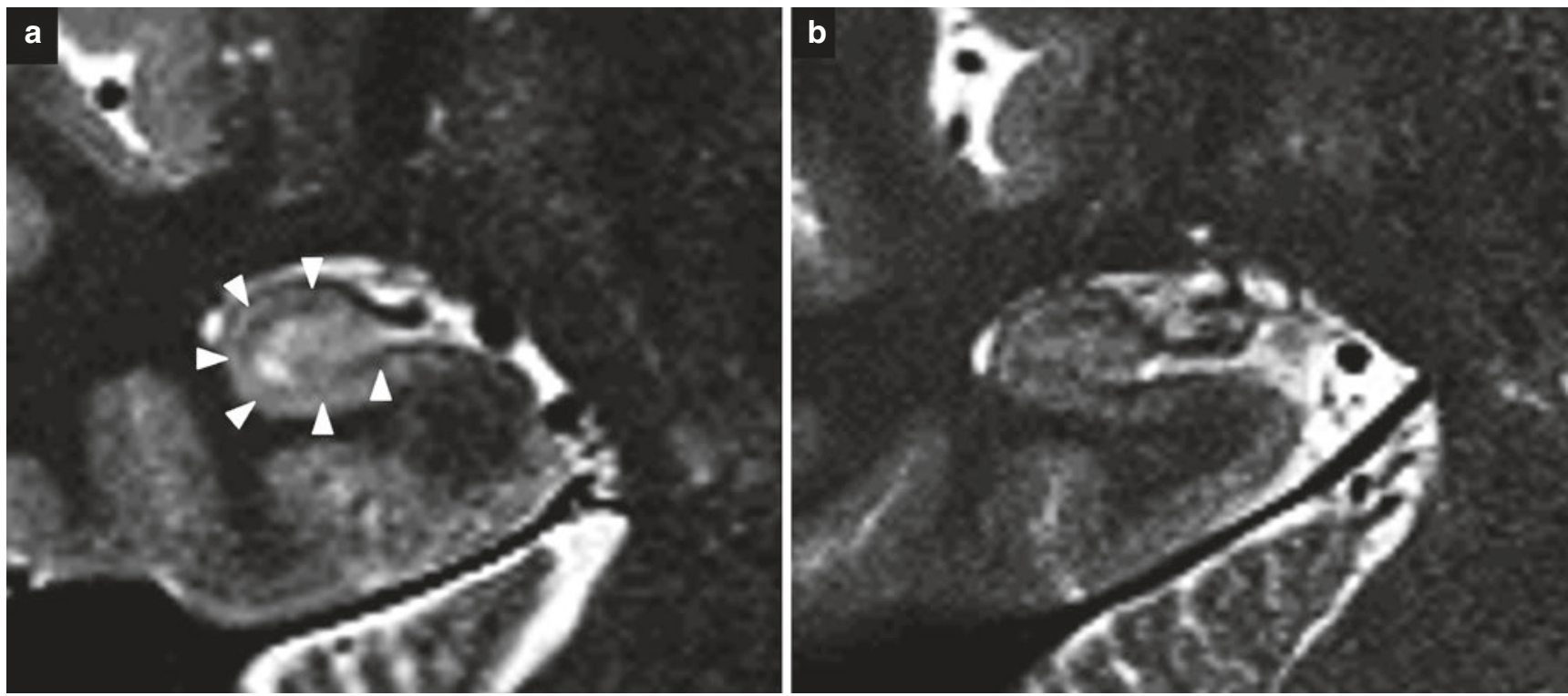

Fig. 10.6 (a, b) Right hippocampus internal architecture: (a) coronal T2 at 3T showing an inner hypointense layer of the hippocampus representing the stratum lacunosum; (b) at 1.5T the ultrastructural detail of the hippocampus is obscured due to the decreased spatial resolution and SNR

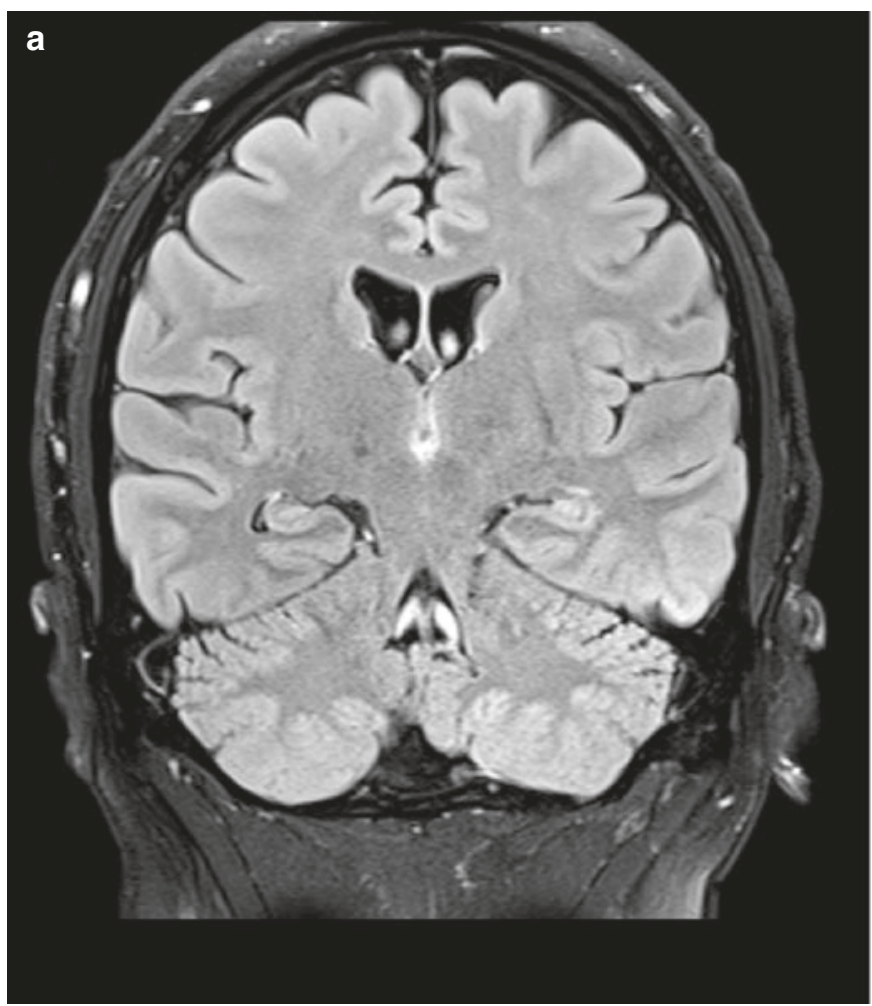

Fig. 10.7 (a, b) Mesial temporal sclerosis (MTS): (a) coronal T2FLAIR shows increased signal in the left hippocampus; (b) axial T1IR demonstrates volume loss in the left hippocampus. The findings

by the subplate (the lowest layer of cortex). Chromosomal mutations, destructive events (ischemia/infections), or toxins may inhibit either of these three processes (proliferation, chemotaxis, or cortical organization) which will lead to abnormalities in stem cell development, migration, or lamination.

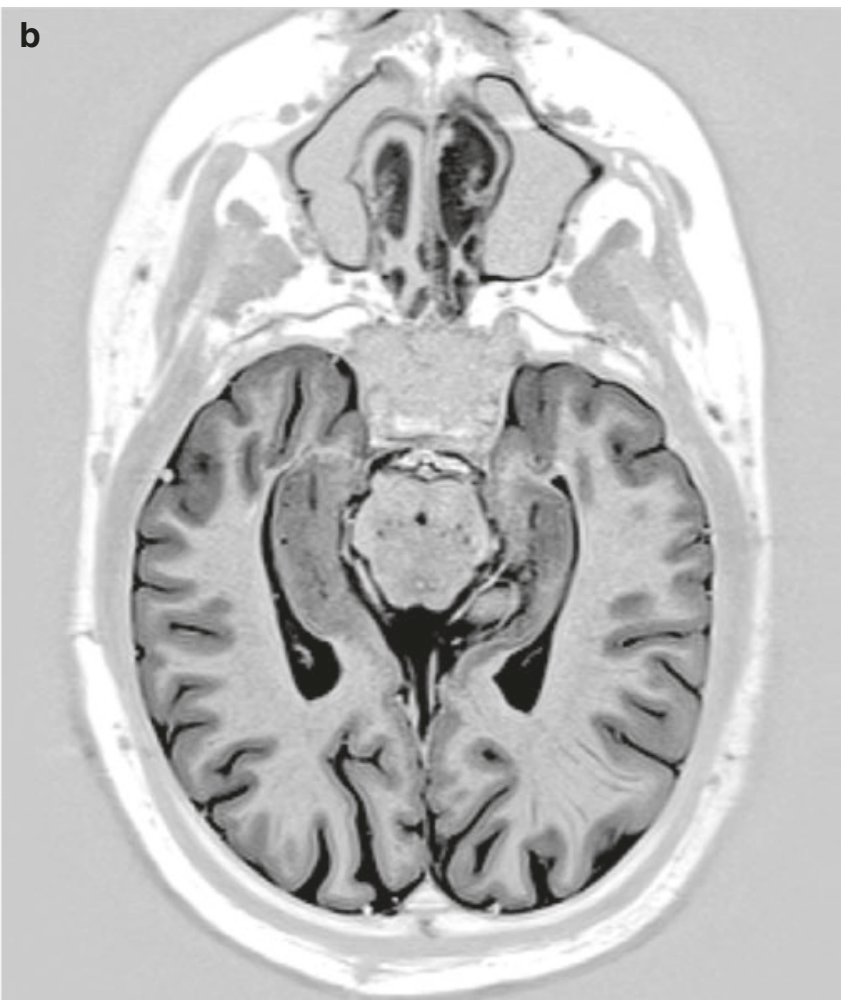

shown are relatively subtle. MRI fails to recognize pathologically detected mesial temporal lobe sclerosis in up to $20 \%$ of the cases

Malformations of cortical development are present in up to $25 \%$ of patients with intractable childhood epilepsy. They are associated with chromosomal alterations, congenital infections, or in utero ischemia. In addition to epilepsy these patients may have developmental delay and focal neurological deficits. 
Malformations related to abnormal stem cell development include the focal or transmantle cortical dysplasias (balloon cell or type II FCDs) and the hemimegalencephalies.

Type II FCD is characterized on histology by dysmorphic neurons with or without balloon cells in addition to cortical dyslamination and are identical to cortical hamartomas in tuberous sclerosis. The transmantle sign is a specific radiologic feature of FCD type II, which is more frequently detected in patients with FCD type IIb than FCD type IIa. Histologically the transmantle sign reflects abnormal cells extending from the ventricle to the cortex manifesting as a linear T2-weighted or FLAIR hyperintensity from ventricle towards the cortex (the radial band or foot) and can be seen in association with a subcortical FLAIR hyperintensity. The abnormal FLAIR hypersignal is again better seen with a narrowed window width. The junction between cortex and white matter is indistinct and the cortical gray matter may be focally thickened (Fig. 10.8).

Signal intensity of the transmantle sign on the T1-weighted sequence could reveal the histologic composition of the type II FCD. Traditionally transmantle sign appears T2-weighted and FLAIR hyperintense and T1-weighted hypointense. More recently a study by Kumura et al. revealed that T1-weighted hyperintense transmantle sign is associated with higher density of balloon cells without significant differences in the number of dysmorphic neurons or the severity of gliosis (Fig. 10.9) [16]. Identifying a T1 hyperintense transmantle sign may have clinical relevance. Although the exact role of balloon cells remains uncertain, some studies have suggested a protective role in reducing spread of the epileptogenic activity.

In hemimegalencephaly a diffuse hamartomatous overgrowth as a result of abnormal stem cell proliferation is present resulting in broad gyri, shallow sulci, and a blurred gray-white matter junction. The ipsilateral ventricle is often enlarged and demonstrates an abnormal straight course of the frontal horn (Fig. 10.10). Clinically, patients present with macrocephaly, hemiplegia, developmental delay, and seizures. The affected hemisphere has no function and thus hemispherectomy can be proposed to these patients. Pre-operative detailed clinical and radiologic assessment is required to determine if there are co-existing abnormalities in the contralateral hemisphere. In addition, diffusion tensor imaging has shown a subset of patients with hemimegalencephaly harbor aberrant midsagittal fiber tracts with intra- or interhemispherically connection which may need to be surgically addressed to improve surgical outcome [17].

Malformations related to abnormal migration are the lissencephalies, the agyria-pachygyrias, and the heterotopias.

In the lissencephalies, there has been a global halt in the migration due to an impaired last phase of neural migration leading to paucity of the gyral and sulcal development with a smooth brain surface and diminished white matter. Patients present with global developmental delay and seizures. Two different types of lissencephaly can be distinguished: the posterior agyria (related to an alteration on Chromosome 17) and the anterior agyria which is an $\mathrm{x}$-linked disease (Fig. 10.11).

Female carriers of the affected X-chromosome present with band heterotopias that is more present in the frontal lobes compared to the parietal lobes. Thus, if females present with band heterotopias, genetic counseling may be indicated as half of their male offspring may be affected by anterior agyria. The band may be thin or thick depending on the amount of arrested neurons during migration. Patients with a thick band have less normal cortex (that can be thinned) and thus present with a more severe developmental delay.

In addition to the "band heterotopia," focal subcortical heterotopia can be present, on imaging, swirling, curvilinear bands of gray matter as well as thinned cortex, and paucity of the white matter are seen. The ipsilateral ventricle may be distorted and there can be an associated callosal hypogenesis.

The third type of heterotopia is called periventricular nodular heterotopia or subependymal heterotopia. On imaging an exophytic smooth ovoid mass in the residual germinal matrix, i.e., along the ventricle is seen (Fig. 10.12). Again, you may have associated anomalies including Chiari malformations, cephaloceles, corpus callosum agenesis, or a Dandy Walker syndrome. In contrast to the other malformations of abnormal migration, the periventricular nodular heterotopia may exhibit quite mild symptoms with normal development and late onset of seizures. If the periventricular heterotopia completely lined the walls of both ventricles, a familiar form has to be considered. Periventricular nodular heterotopia can have different inheritance patterns. Specifically, mutation in the FLNA gene is inherited in an X-linked dominant pattern with male offspring experiencing more severe symptoms of the disorder than females, and in most cases die before birth. Hence genetic counseling for female patient of reproductive age with extensive periventricular heterotopia may be considered.

Malformations related to abnormal cortical organization encompass polymicrogyria, schizencephaly, and FCD type I (non-balloon cell). In polymicrogyria, neurons reach the cortex but distribute abnormally, thus multiple small gyri are formed (Fig. 10.13). Polymicrogyria is most commonly found around the posterior Sylvian fissures, when bilaterally present in the perisylvian region patients present with pseudobulbar palsy.

In open-lip schizencephaly a cleft that is lined by gray matter reaches from the periphery to the ventricle, while in the closed-lip schizencephaly gray matter is reaching from the periphery to the ventricle and a dimple is seen in the ventricular wall. Schizencephaly can be 
multifocal and bilateral. The cortex lining the defect is polymicrogyric with ill-defined margins to the white matter. Finally, FCD type I (non-balloon cell) is a disorder of lamination. Imaging features are very subtle and only mild focal blurring of the gray-white matter junction may be present. This type of dysplasia is often undetectable on MRI.

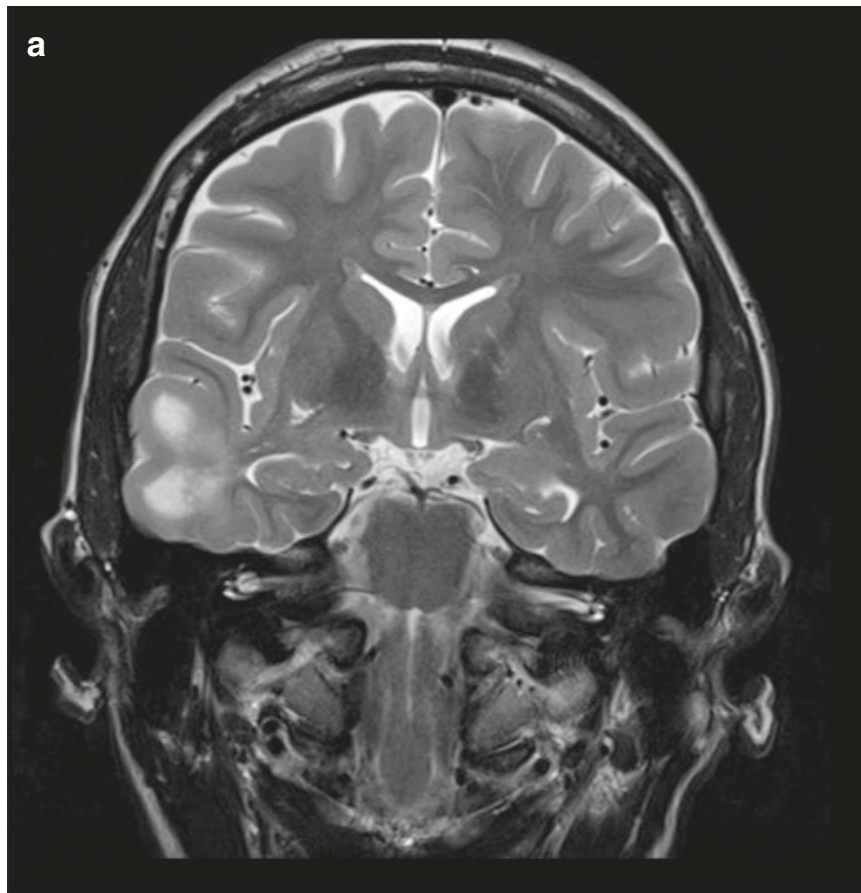

C

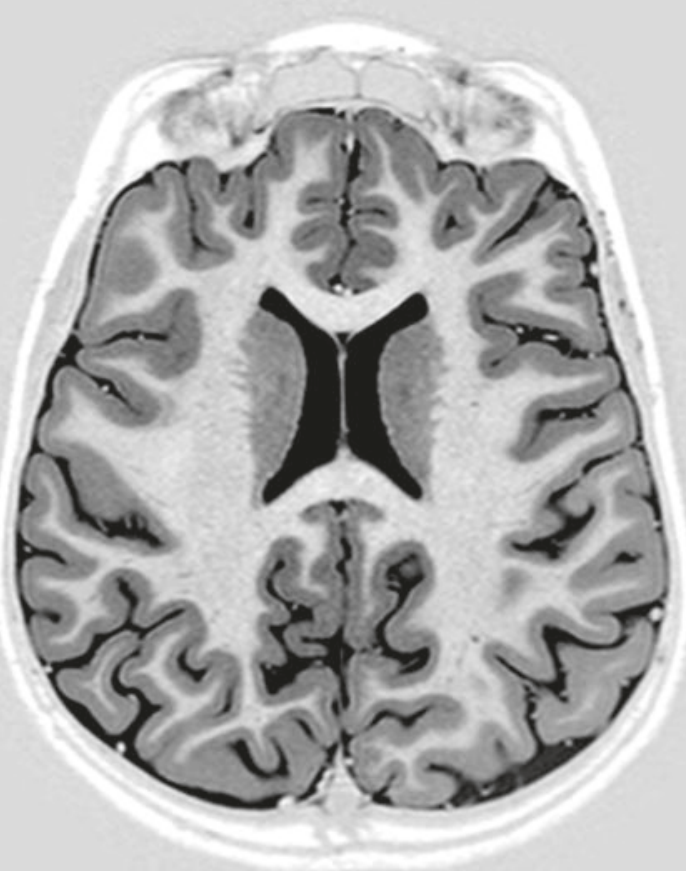

Fig. 10.8 Three cases of FCD type II. (a, b) patient 1; (a) coronal T2 with large subcortical area in the right temporal lobe with prolonged T2. The increased signal stretches in to the temporal horn of the right ventricle; (b) coronal T1 with gadolinium. The corresponding area has decreased signal on T1-weighted image. No enhancement. (c, d) patient 2; (c) axial T1IR with very subtle signal changes at the bottom of a

\section{Key Point}

- Malformations of cortical development are commonly seen in pediatric patients with medication refractory epilepsy. Often these patients have some form of cognitive impairment or developmental delay.
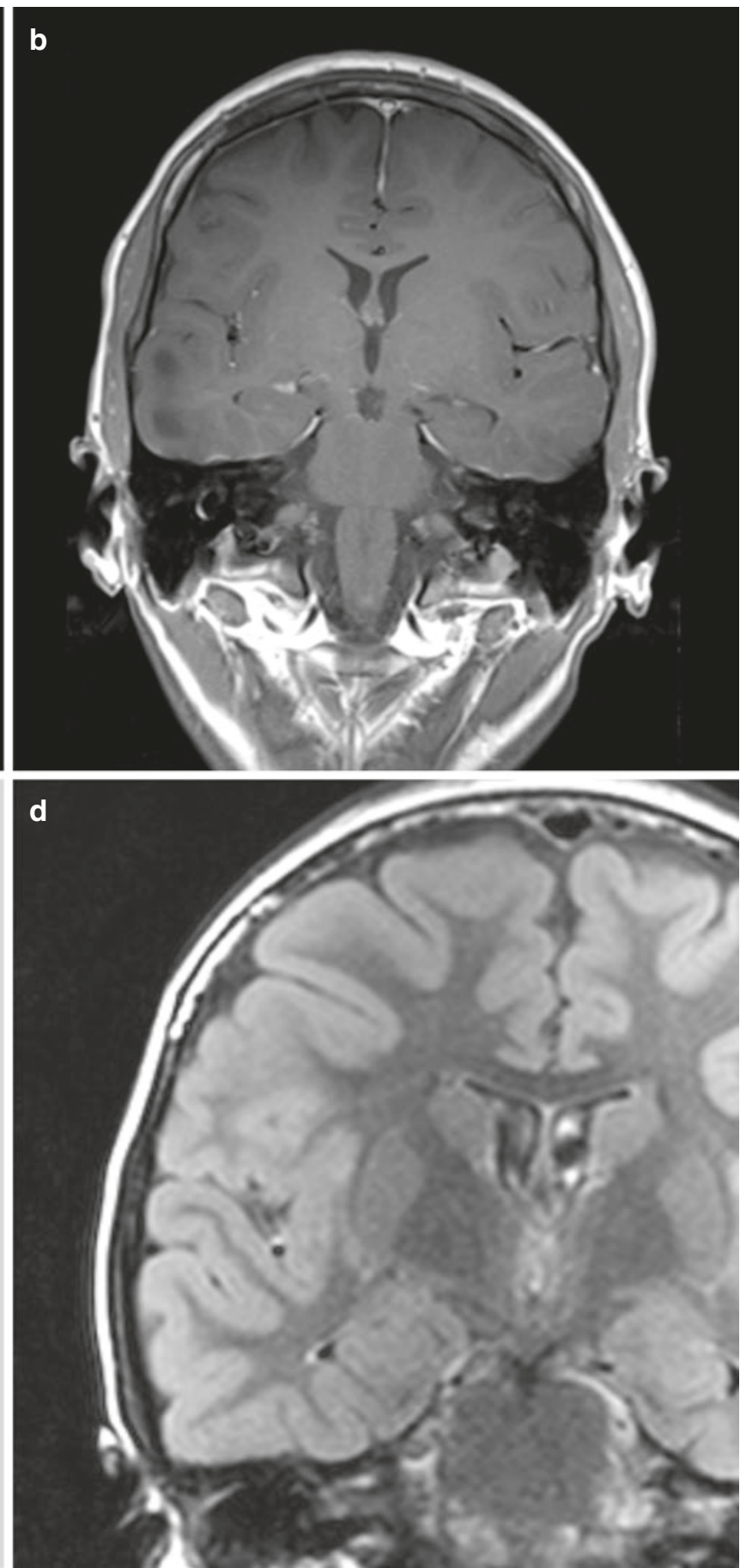

sulcus lateral in the frontal lobe; (d) coronal T2FLAIR shows increased signal in the same are with a faint band stretching towards the lateral ventricle. (e) patient 3, boy, 3 months old, axial T1IR shows a region with thickened cortex in the right frontal lobe. Notice the premature myelinization of the white matter tracts involved in the seizures 
e

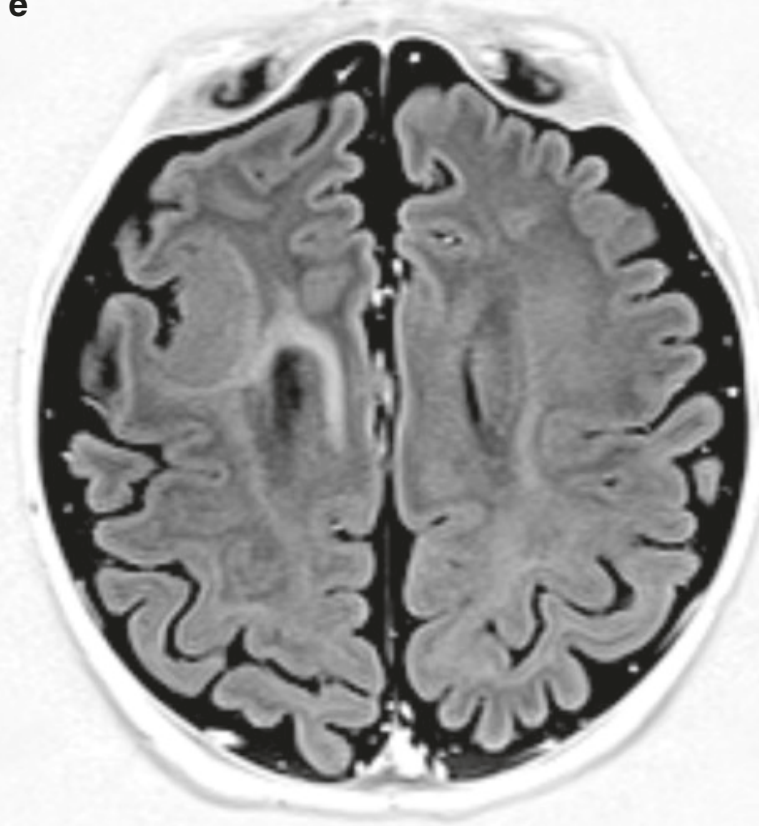

Fig. 10.8 (continued)

\subsubsection{Epileptogenic Tumors}

While virtually all tumors may cause epilepsy, there are certain tumors that have a very high propensity of eliciting medication refractory seizures. As most of these are benign and just by means of location (i.e., within the cortical-white matter interface and with temporal lobe predilection) cause the seizures, these are often very good candidates for surgery. As a general discussion of all tumors is beyond the scope of this chapter, we will focus only on three tumors that are commonly associated with seizures: the gangliogliomas, the DNETs, and the tuber cinereum hamartomas.

Gangliogliomas are cortically based, partly cystic tumors that may calcify and that harbor an enhancing nodule (Fig. 10.14). Gangliogliomas occur in young adults and older children, when present under the age of 10 they are often larger with more cystic components. They are mainly located in the temporal lobes but can also occur in parietal and frontal lobes. Cortical dysplasias (coined type III) can be associated with a ganglioglioma. Differential diagnoses for gangliogliomas are DNETs, pilocytic astrocytomas, pleomorphic xanthoastrocytomas, gliomas, and neurocysticerco-

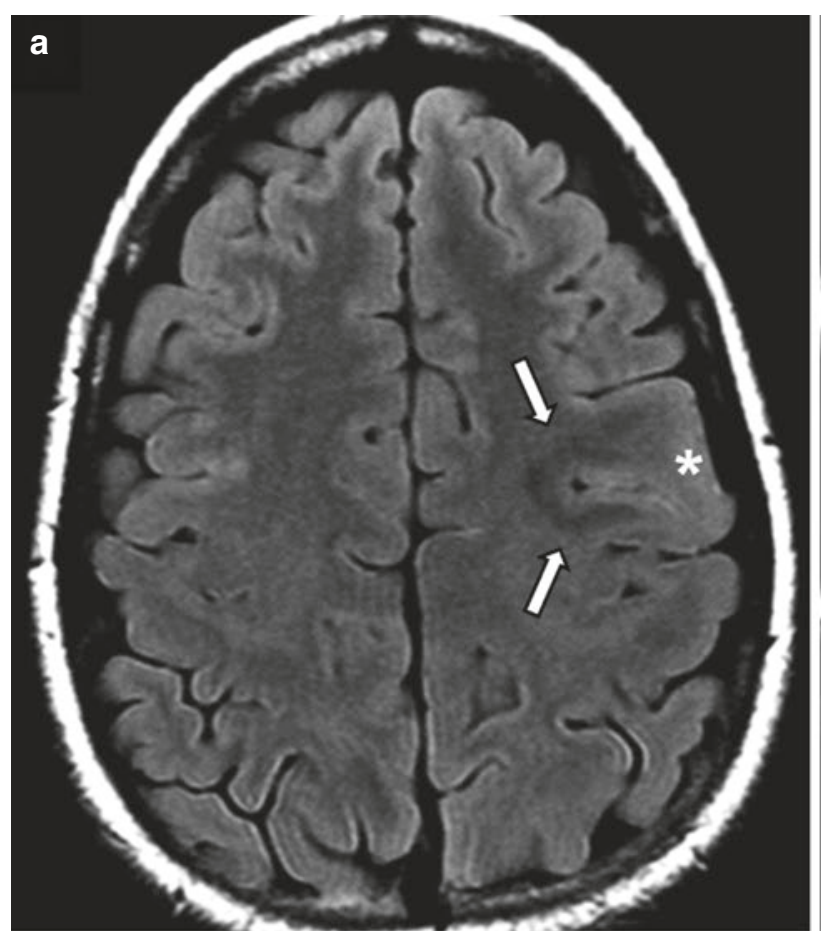

Fig. 10.9 Left frontal type II FCD. (a, b) axial and coronal FLAIR images demonstrate focal gyral thickening posteriorly in the left frontal gyrus with an associated curvilinear hypointense band following the

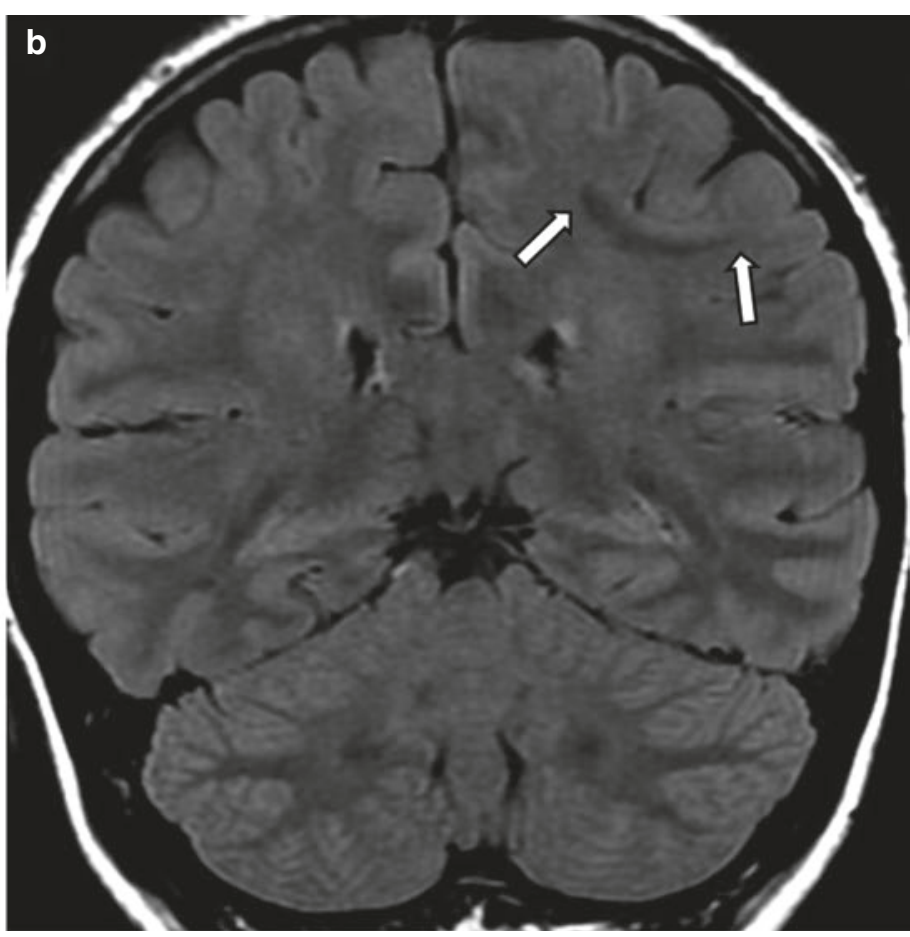

bottom of the sulcus. (c) T1 inversion recovery reveal hyperintensity in this area 


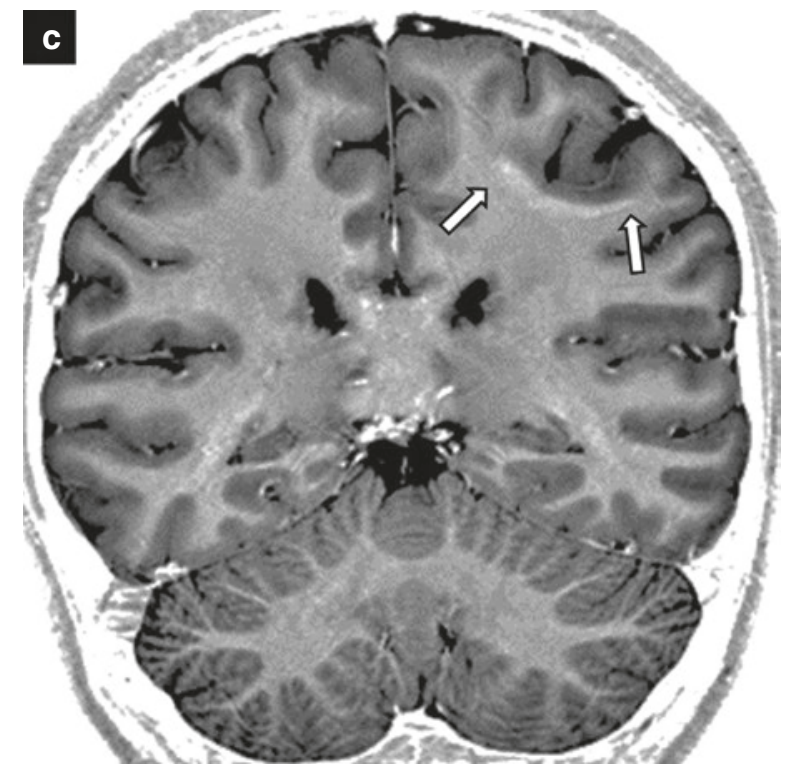

Fig. 10.9 (continued) sis. In adults, solid appearing ganglioglioma may mimic high grade glioma and advanced imaging techniques such as MR perfusion maybe a useful tool for pre-operative evaluation (Fig. 10.15).

DNETs are well demarcated, bubbly, intracortical masses that also are most common in the temporal, parietal, and frontal lobes (Fig. 10.16). They may calcify and enhancement is very rare and if present should lead to more intensive follow-up as the enhancing portion of a DNET may recur following surgery. Top differential diagnoses for DNETs are cortical dysplasia, ganglioglioma, pilocytic astrocytoma, glioma, neuroepithelial cysts, and dilated VR spaces.

Tuber cinereum hamartomas present with the combination of gelastic seizures and precocious puberty. They are located at the floor of the third ventricle (i.e., the tuber cinereum) do not enhance and are isointense to cortex (Fig. 10.17). They are non-neoplastic tumors with disorganized collection of neurons and glia.
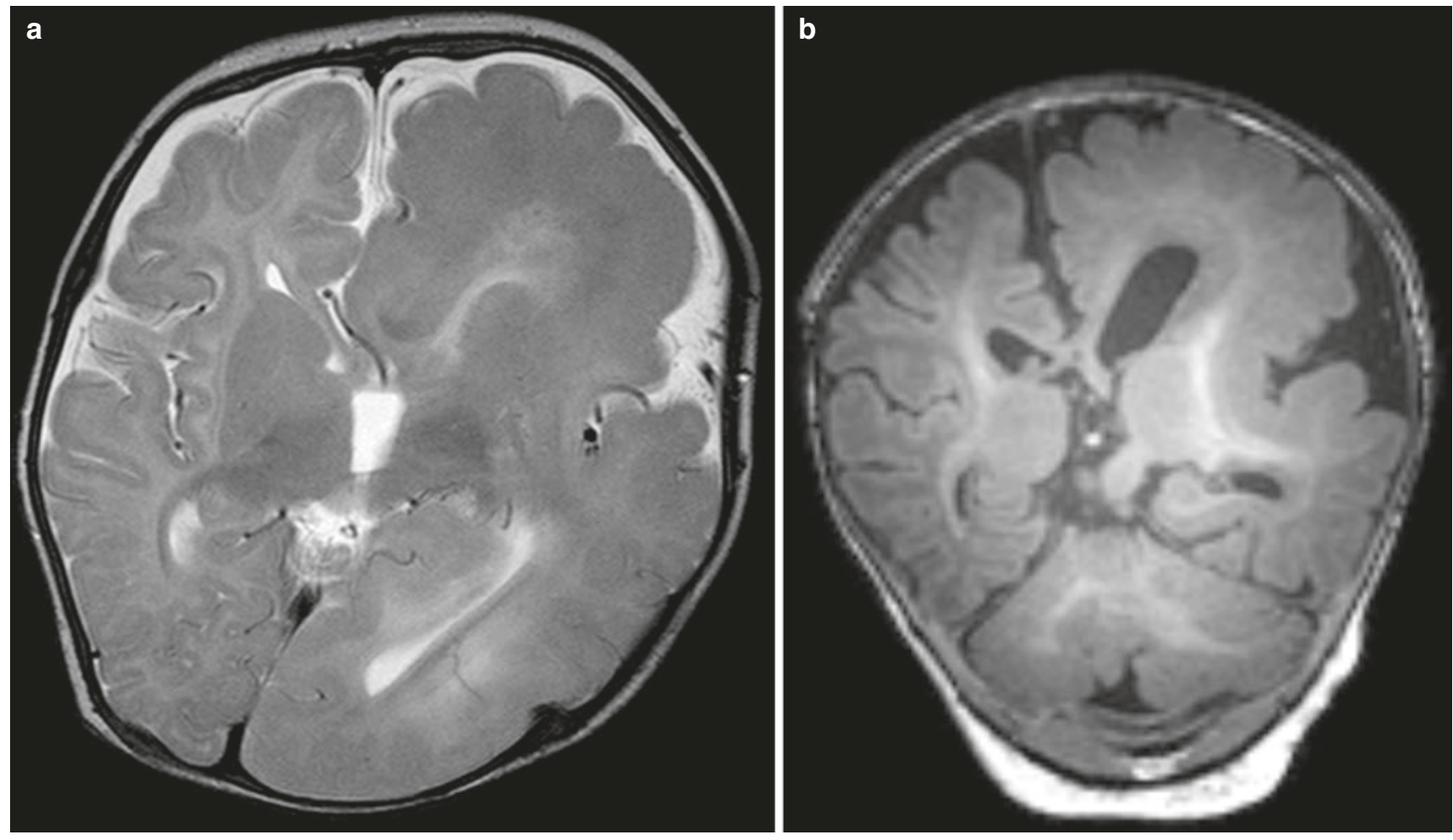

Fig. 10.10 (a, b) Boy, 6 months. Hemimegalencephaly. (a) axial T2; (b) coronal T1. The left hemisphere is enlarged with broad gyri and shallow sulci. The ipsilateral ventricle is enlarged with an abnormal

shape of the frontal horn. Indistinct gray/white matter is noted throughout the entire hemisphere 

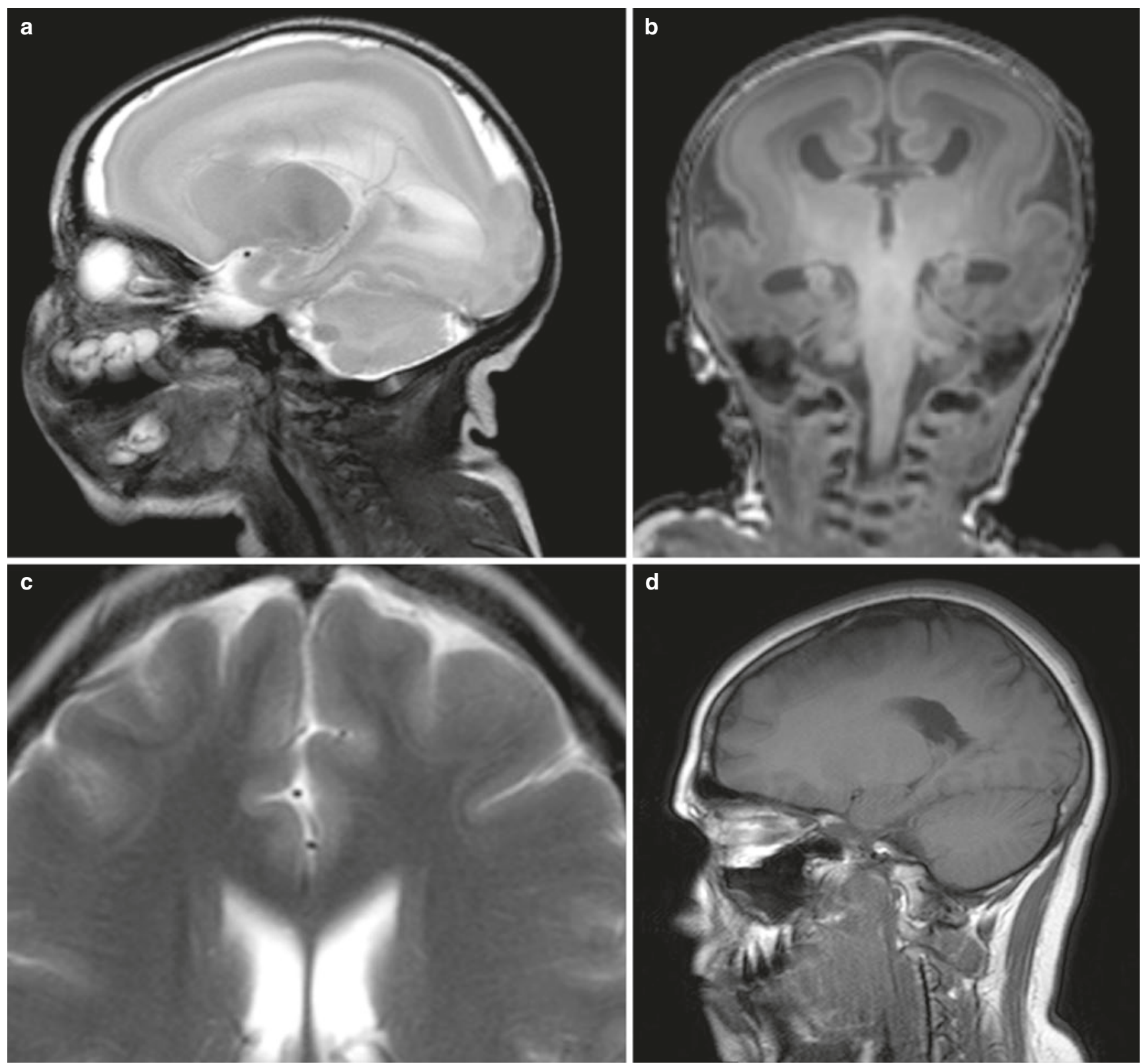

Fig. 10.11 (a, b) X-linked lissencephaly, boy, 2 weeks old. (a) sag t2; (b) coronal $\mathrm{T} 1$. Lissencephaly with agyria more pronounced in the anterior part of the brain. In addition, callosal hypogenesis. (c, d) The mother of the boy in (a, b) female carrier. (c) Axial T2 (detail); (d) coronal T1; Subtle subcortical band heterotopia in both frontal lobes

\subsubsection{Miscellaneous: Vascular Malformations/ Trauma/Infection/Phakomatoses}

Similar to the previous paragraph, it is beyond the scope to in detail describe imaging features of vascular malformations, infections or trauma that can go along with seizures and most of the entities are described in other chapters of this syllabus. We therefore only want to highlight few epilepsy-relevant facts and features of these miscellaneous conditions.

Brain AVMs can cause seizures due to previous hemorrhage and scarring, hemosiderin deposition (especially when close to the cortex), or gliosis. AVMs in the temporal lobe have a higher likelihood of producing seizure due to interference of the normal blood supply and drainage of potentially epileptogenic structures such as the hippocampus.

While cavernomas that are deeply located in the white matter rarely cause seizures, those that are cortically located and have hemosiderin staining reaching the cortex, and in particular the mesial temporal lobe structures, are very often associated with seizures as the hemosiderin stain is believed to have a strong irritative potential for neurons. They are best visualized on T2 gradient echo or SWI sequences where they 

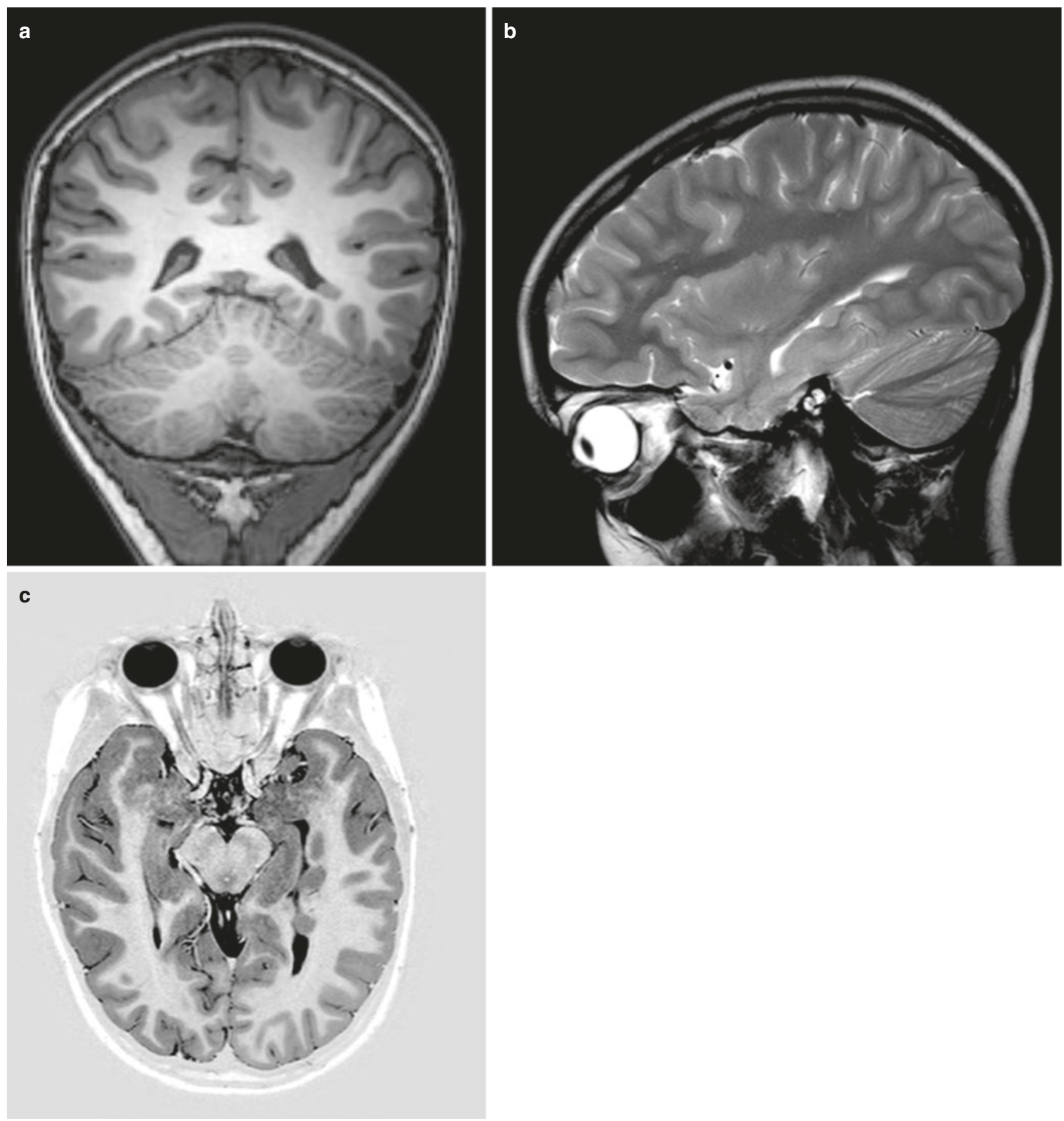

Fig. 10.12 (a-c) Periventricular nodular heterotopia: (a) coronal T1; (b) sagittal T2; (c) axial T1IR. Well delineated smooth ovoid masses lateral to the trigone and temporal horn of the left ventricle. Note that the signal is identical to that of cortex in all sequences

demonstrate with the classical blooming artifact (Fig. 10.18). Cavernomas may be multiple and they can be associated with developmental venous anomalies (DVA). New intracavernomatous thrombosis or hemorrhage may lead to change in seizure frequency.

Patients with previous trauma can experience posttraumatic seizure disorder, especially after having sustained con- tusional hemorrhages of their temporal lobes as gliosis and hemosiderin staining can cause irritation of the surrounding cortex.

Neonatal anoxic ischemia or hypoxemia can cause ulegyria-i.e., a scar/defect of the cerebral cortex that mainly involves the cortex in the depth of the sulcus whereas the cortical crowns remain relatively unaffected. This peculiar pattern can be 

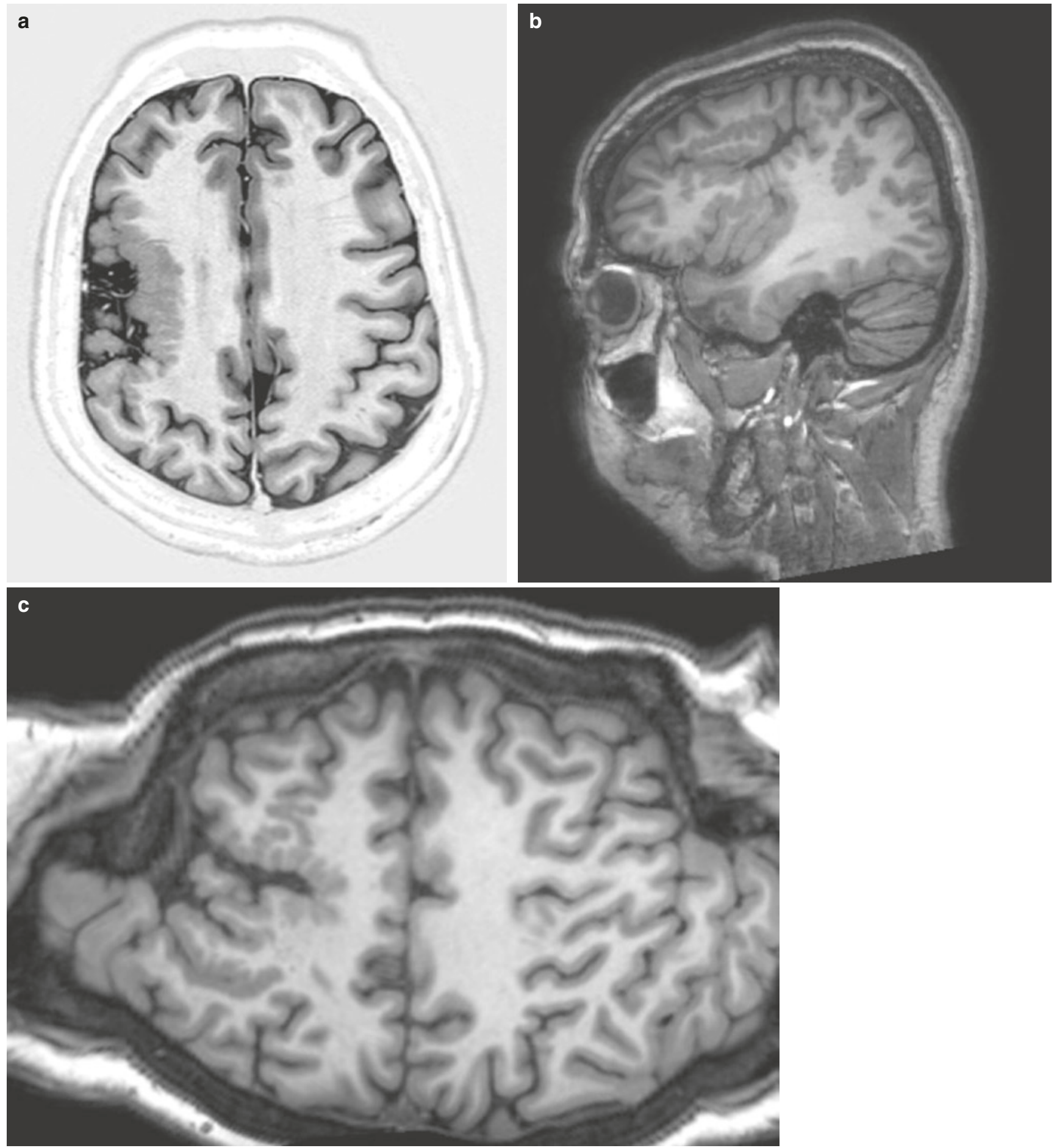

Fig. 10.13 (a-c) Two patients with polymicrogyria. (a) Axial T1IR; (b): sagittal T1. Patient 1. Abnormal gyration in the right hemisphere with a large region with polymicrogyria. (c) Patient 2, "pancake view" from a 3D T1-sequence gives a very good overview of the migration anomalies in the right hemisphere. It also increases the chance to detect subtle changes - see small area with polymicrogyria in the left hemisphere! 

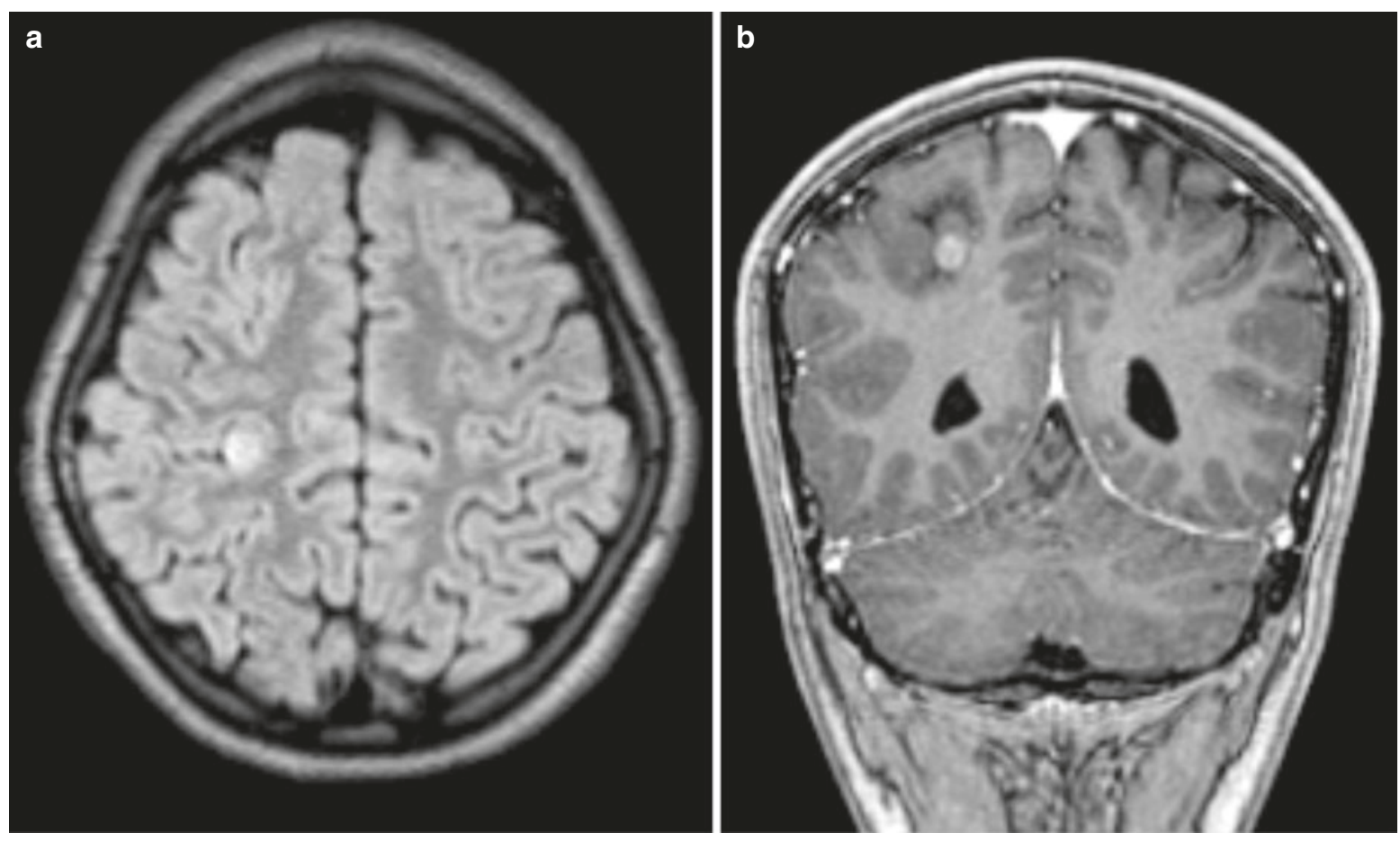

Fig. 10.14 (a, b) Ganglioglioma close to the right postcentral sulcus. (a) axial T2FLAIR with a small, cortical/subcortical, nodular high-signal area in the right parietal lobe close to the postcentral sulcus; (b) coronal T1 with gadolinium shows contrast enhancement in the nodulus
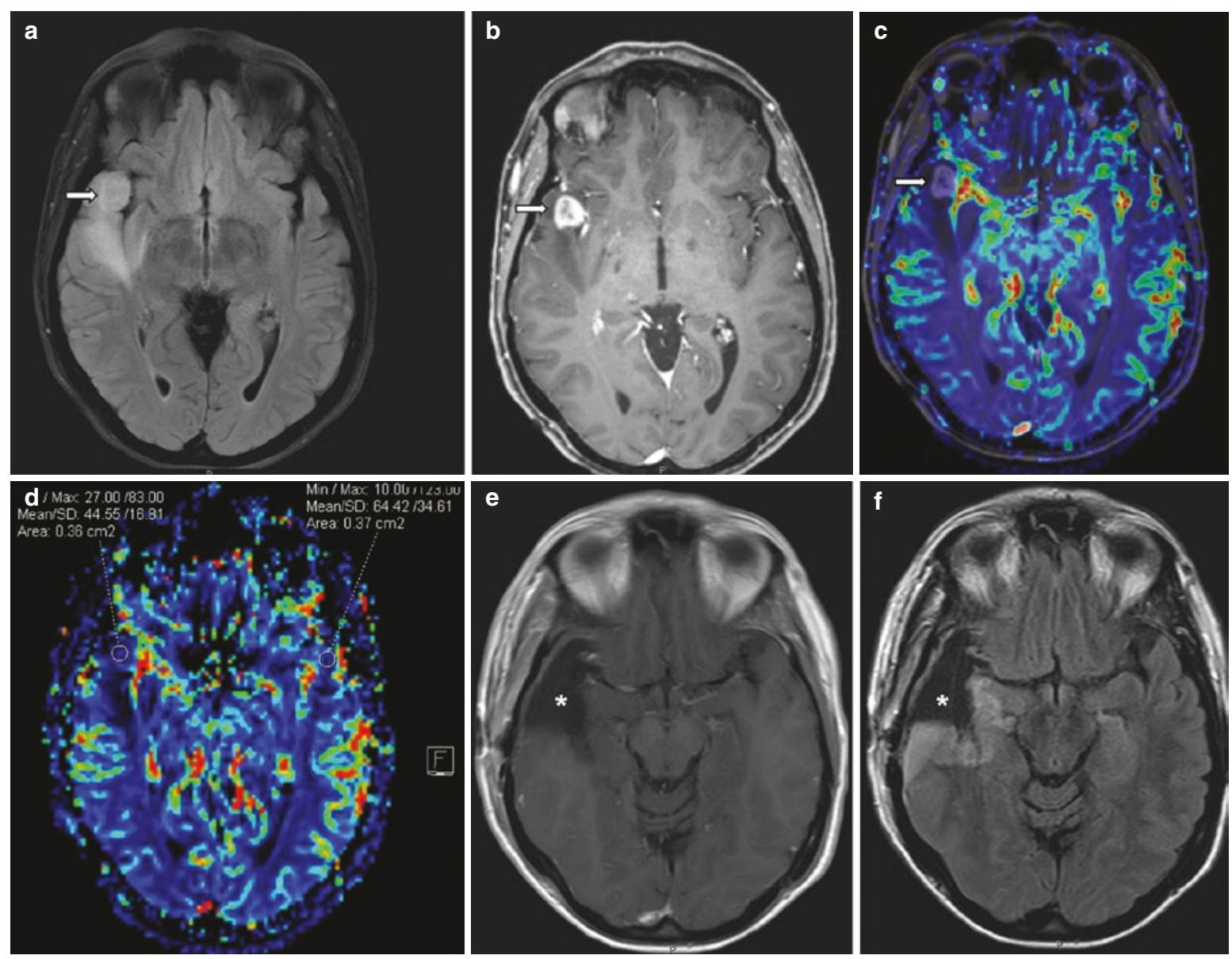

Fig. 10.15 Right superior temporal gyrus ganglioglioma in a middleaged patient. Pre-operative MRI (a, b) axial T2FLAIR and post gadolinium enhanced axial T1 images showed an avidly enhancing cortically based mass with moderate peri-lesional edema. (c, d) Despite the avid lesional enhancement, MR perfusion showed low rCBV value consistent with a low-grade glioma. (e, f) Diagnosis of ganglioglioma was confirmed following mesial sparing temporal lobectomy 

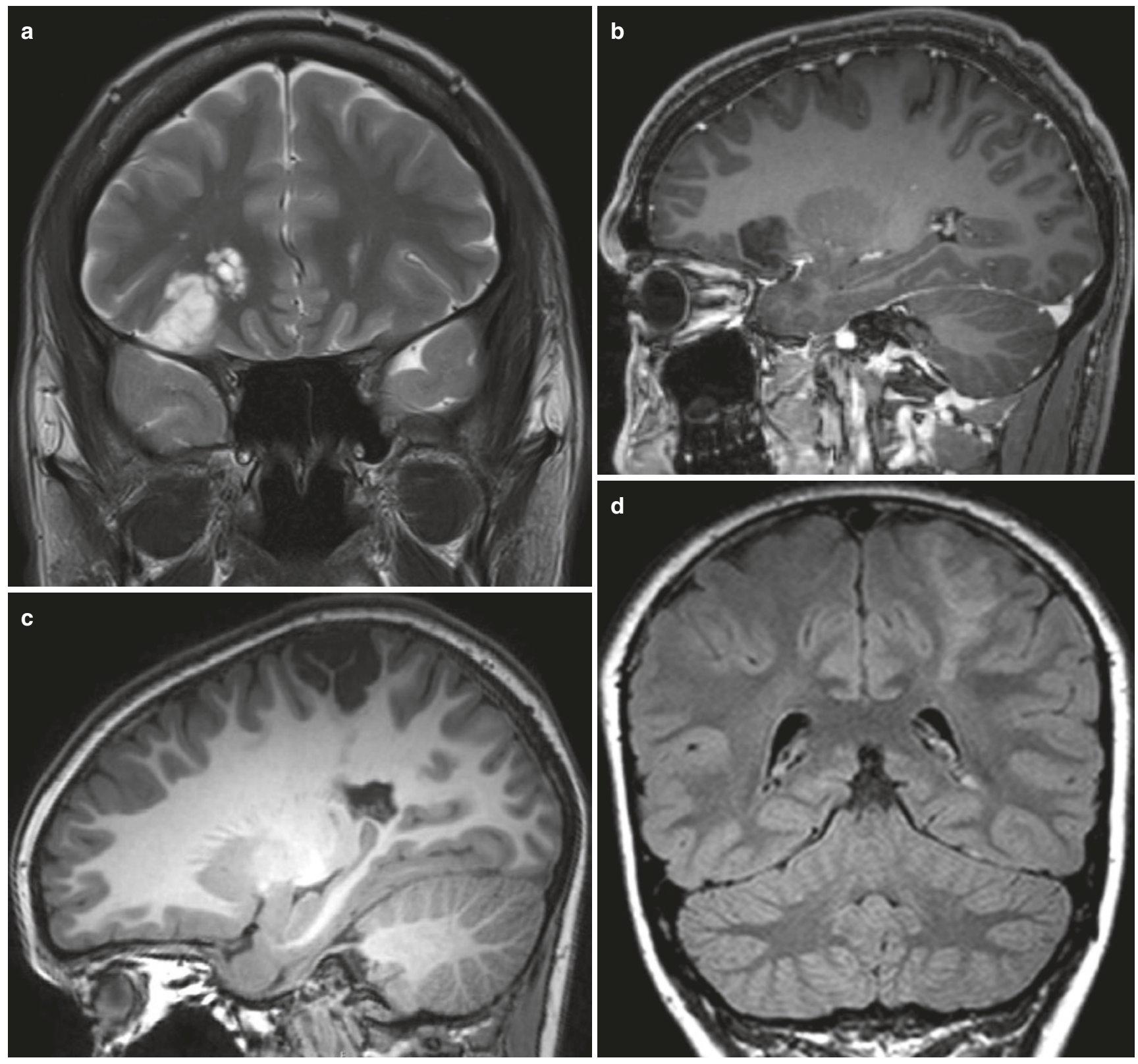

Fig. 10.16 Two patients with DNET. (a, b) Patient 1. (a) coronal T2; (b) sagittal T1 with gadolinium; well delineated cortical/subcortical bubbly mass in the right frontal lobe with prolonged $\mathrm{T} 2$ and no contrast enhancement typical of a DNET. (c, d) Patient 2: (c) sagittal T1; (d)

explained by the vascular supply of the gyri in the newborn that leads to a better perfusion of the apices of the gyri as compared to the depth of the sulci. There will be paucity of the white matter and, as the lesion occurred prior to complete myelination, a relatively mild gliosis. If the perinatal ischemia has only involved one hemisphere (perinatal stroke) a Dyke Davidoff Mason syndrome will ensue where stable hemiatrophy is present with hypertrophy of the skull and the sinuses, paucity of white matter, ventricular enlargement, and mild gliosis.

Virtually any infection (bacterial, fungal, parasitic) can produce epileptogenic lesions, and world-wide, infections are coronal T2 FLAIR; This DNET in the left parietal lobe is associated with a FCD type II. Notice the streak with signal changes which stretches towards the lateral ventricle

the leading cause of epilepsy. A typical example is neurocysticercosis which is a very common cause of focal epilepsy in the developing world. In the early vesicular, colloidal or granular nodular stages, the ictal focus is likely to originate from the cysticercus zone. Recurrent seizures might cause hippocampal damage or dysfunction. In the late nodular calcified stage the cysticercus zone becomes less active and but damages to the mesial temporal structures may lead to acquired MTS which becomes the new ictal focus (Fig. 10.19).

Rasmussen's encephalitis is a presumably autoimmune mediated chronic inflammation of the brain that presents 

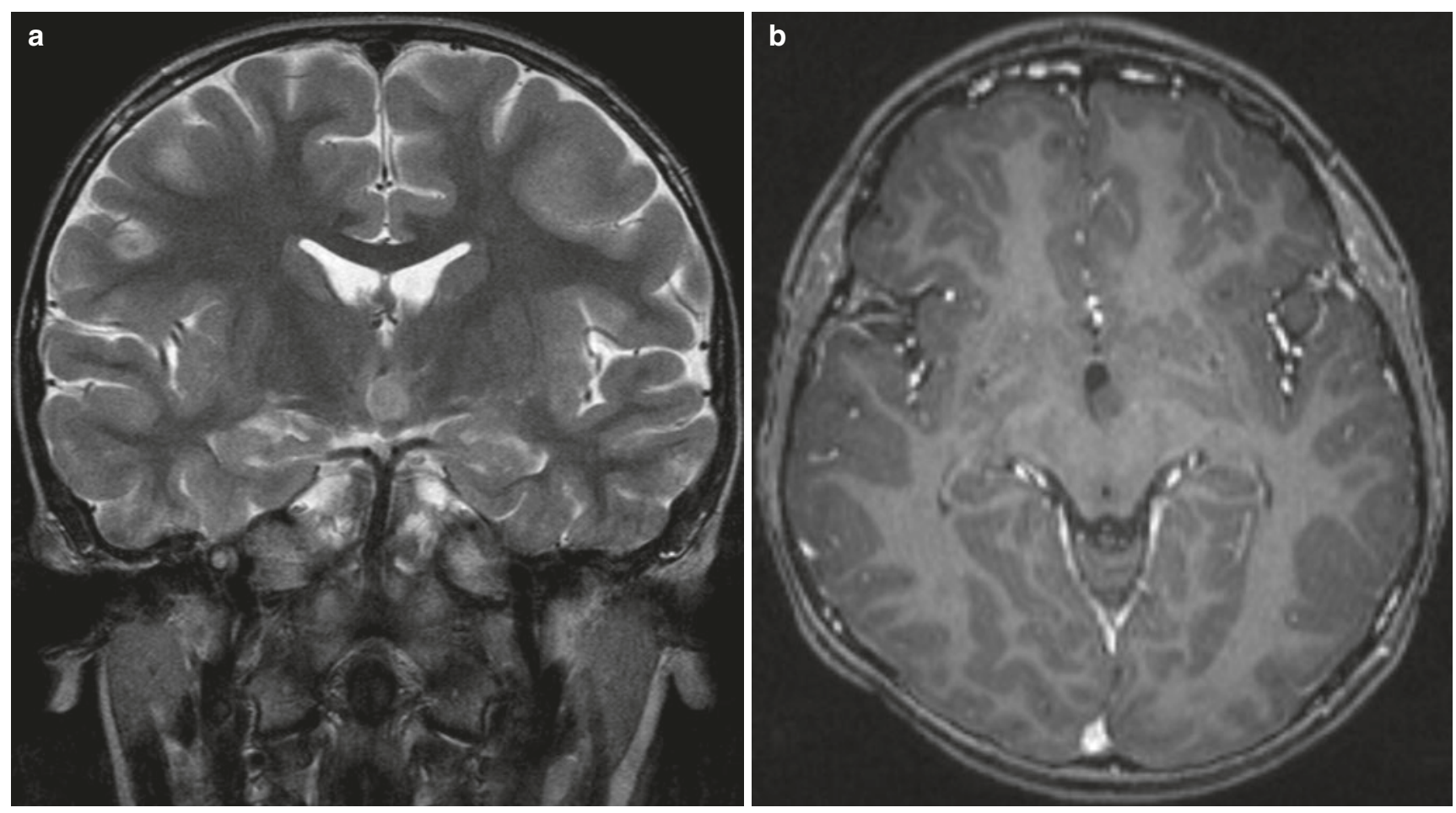

Fig. 10.17 (a, b) Hypothalamic hamartoma. (a) Coronal T2 with a lobular mass close to the left wall of the third ventricle. (b) Axial T1 with gadolinium detects no contrast enhancement in the mass
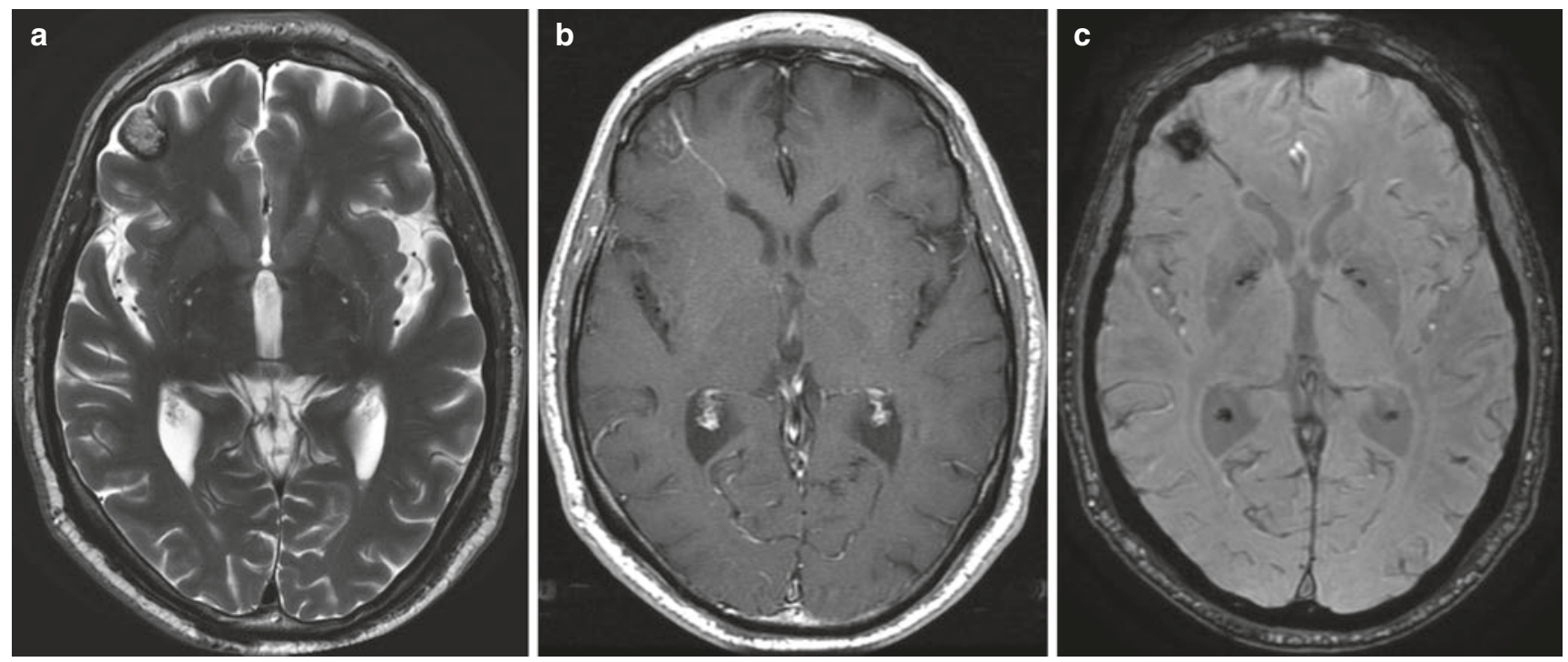

Fig. 10.18 (a-c) Cavernoma in the right frontal lobe with an associated DVA. (a) axial T2 shows the superficial lesion with heterogenous signal; (b) axial T1 with gadolinium shows an associated vascular

with progressive gliosis and volume loss. Patients experience seizures and a progressive hemiparesis.

Antero-basal temporal lobe encephaloceles are lesions that are either related to a congenital defect of the bone or to previous trauma. Brain tissue can extend into the pterygopalatine fossa through the bony defect at the base of the structure, a DVA; (c) axial SWAN-sequence. This susceptibility sensitive sequence shows the classical blooming artifact of the blood products within the cavernoma

greater sphenoid wing in the region of the foramen rotundum and pterygoid process. The herniated brain demonstrates high T2/FLAIR signal and is believed to be the epileptogenic focus. Following resection of the abnormal brain tissue seizure freedom can be obtained in a very large proportion of cases. 

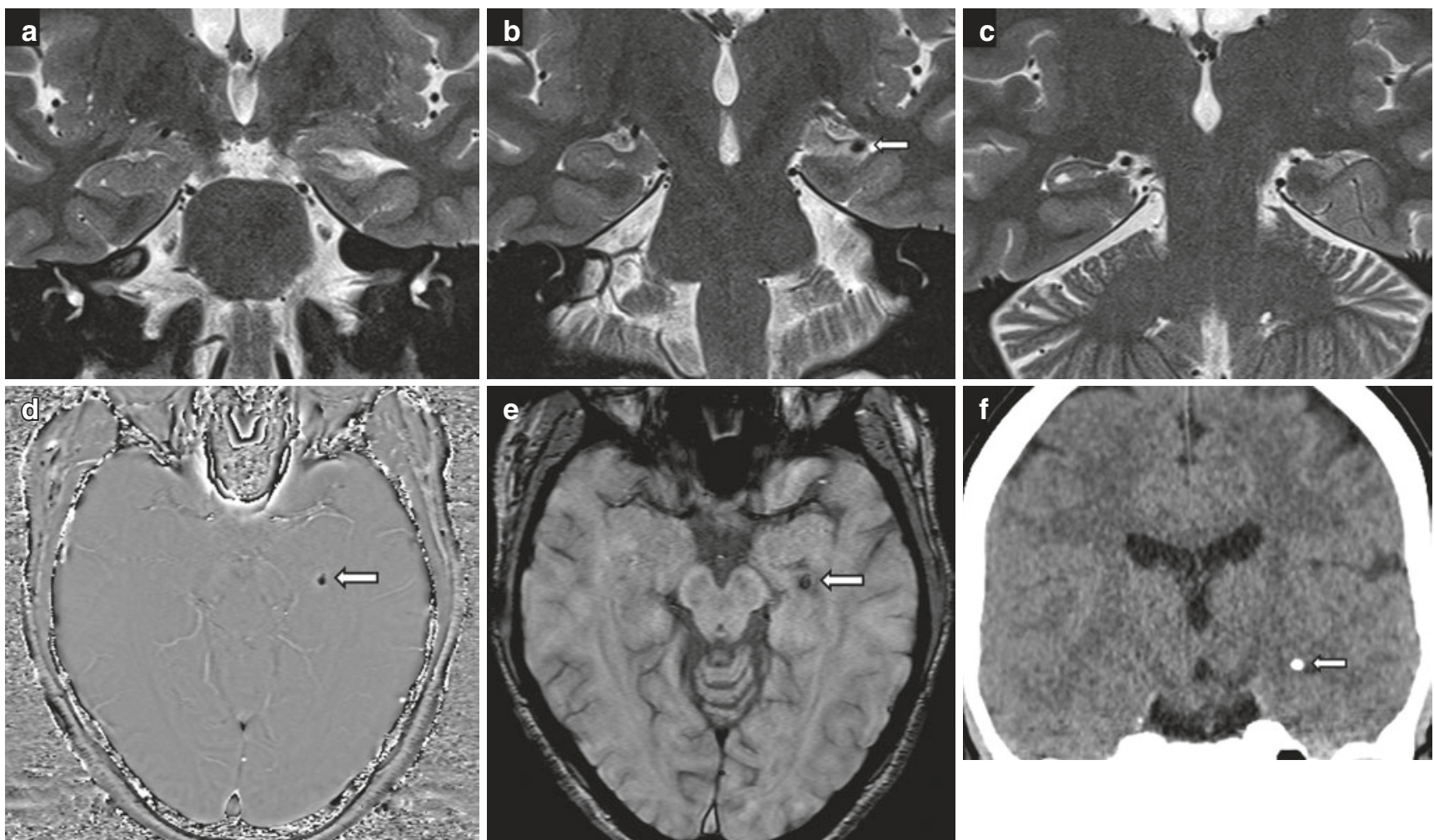

Fig. 10.19 Nodular calcified neurocysticercosis in the left hippocampus leading to acquired MTS. (a-c) Coronal T2 images show a small circumscribed hypointense lesion in the left hippocampal head with associated significant volume of the left hippocampus and obscuration of the internal hippocampal architecture. (d, e) SWI and phase image show positive phase shift suggestive presence of calcification. (f) CT scan confirms the calcification
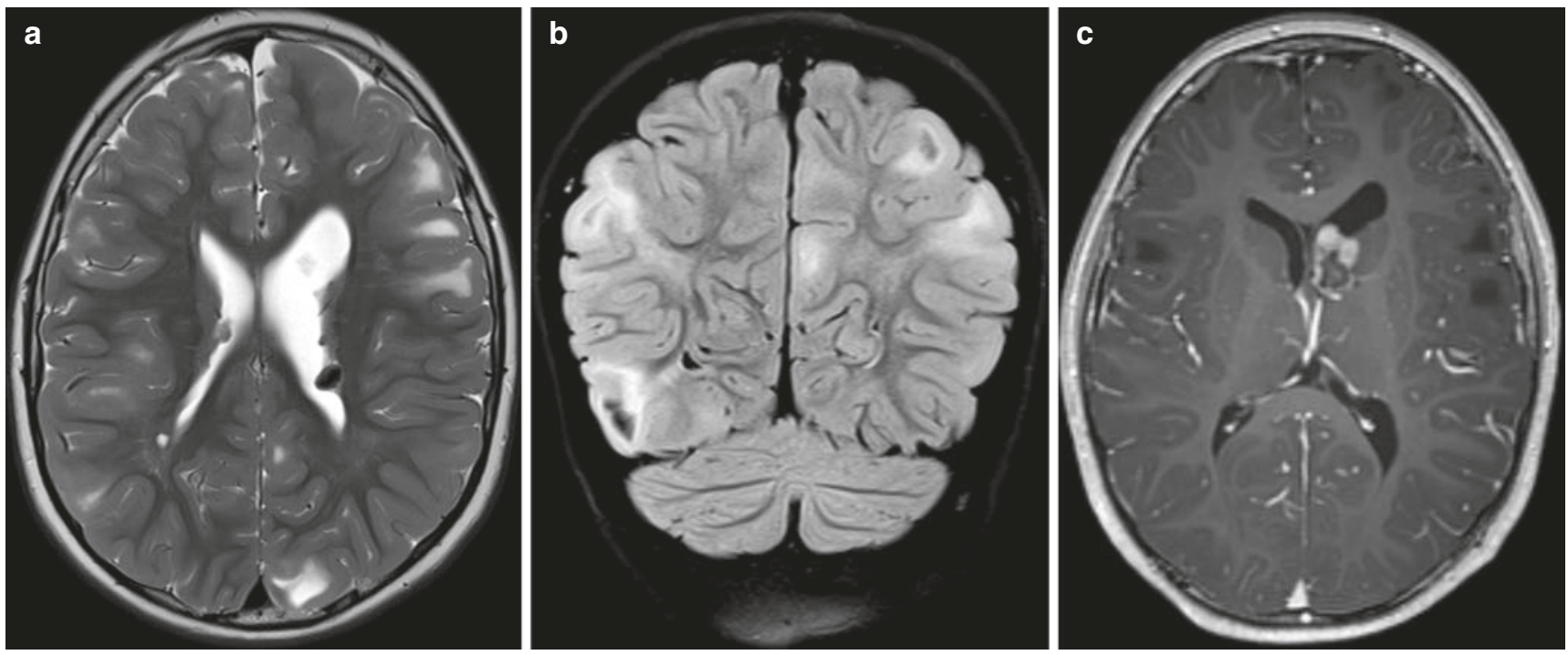

Fig. $10.20(\mathbf{a}-\mathbf{c})$ Tuberous sclerosis; (a) axial T2 and (b) coronal T2FLAIR show subependymal hamartomas and widespread cortical and subcortical signal changes; (c) axial T1 with gadolinium with a large giant cell astrocytoma in a classical position, close to the foramen of Monroe in the left lateral ventricle 

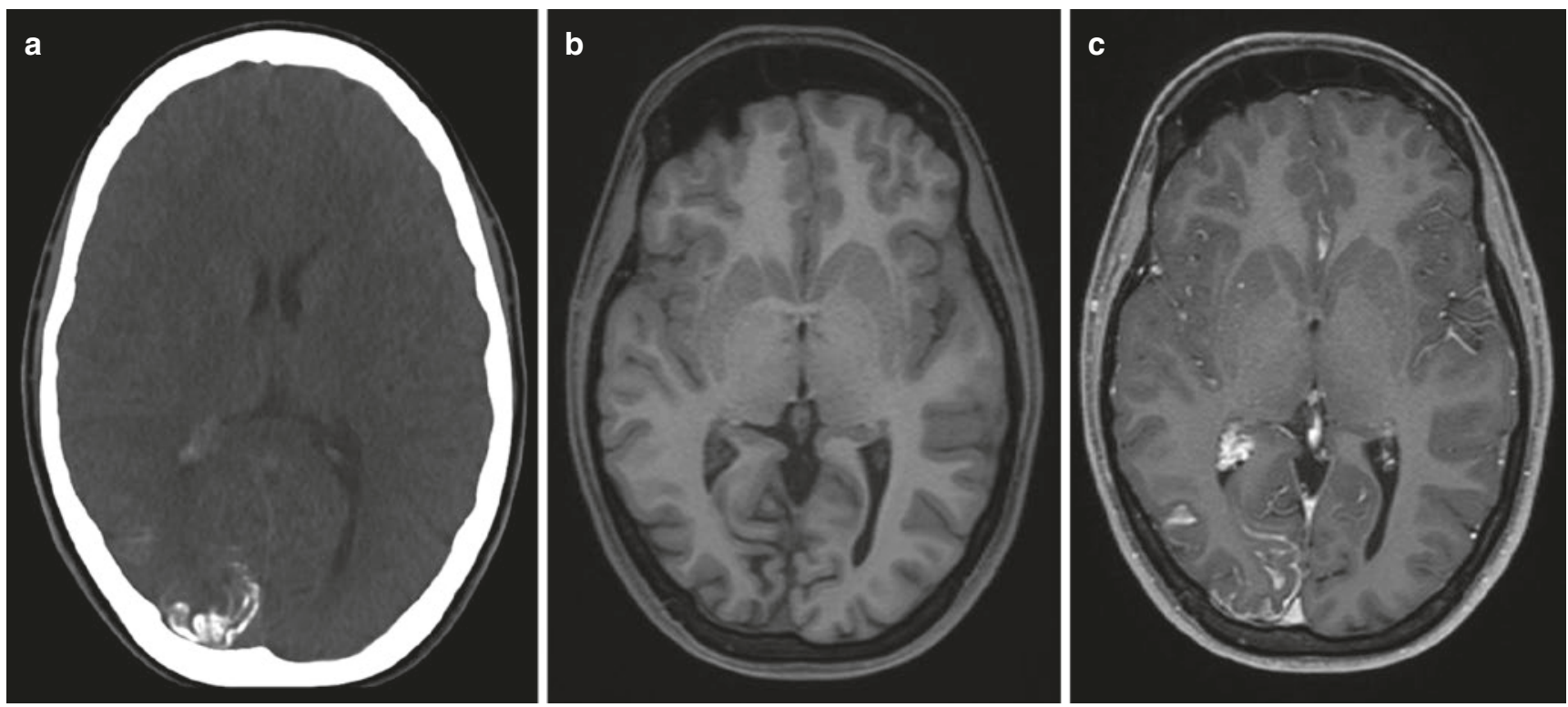

Fig. 10.21 (a-c) Sturge-Weber syndrome; (a) axial CT shows curvilinear cortical calcifications in the right occipital lobe; (b) axial T1 without and (c) with gadolinium shows contrast enhancement caused

The two phakomatoses commonly associated with seizures are tuberous sclerosis and Sturge-Weber syndrome. In tuberous sclerosis, multiple hamartomas are present within the cortical/subcortical region (Fig. 10.20). These are similar in histology to the FCD type II and are therefore believed to be epileptogenic. In addition, patients may develop subependymal calcification as well as a subependymal giant cell astrocytoma; however, the latter two lesions are not believed to be epileptogenic. In Sturge-Weber syndrome the cortical calcification as well as the pial angiomatosis along the cortex is presumably related to the seizures. In addition, patients may present with choroid plexus hypertrophy and brain hemiatrophy as well as a facial port-wine stain (Fig. 10.21).

\section{Key Point}

- Many other pathologies including tumors, vascular malformations, phakomatoses, or remote infections can cause medication refractory epilepsy especially if the structures involved are close to the mesial temporal lobe structures.

\subsection{Conclusion}

Neuroimaging in patients with refractory epilepsy will find abnormalities in as high as $85 \%$ of cases and therefore plays a crucial role in the identification of epileptogenic lesions and their possible surgical removal. A dedicated epilepsy protocol is necessary to identify these lesions and the MR

by pial angiomatosis. Note also hypertrophy of the ipsilateral choroidal plexus, typical for this phakomatosis

should be interpreted in conjunction with EEG, MEG, neuropsychological testing, and clinical semiological data to increase the likelihood of identifying these often very subtle lesions.

\section{Take Home Messages}

- When evaluating a dedicated seizure protocol MR, a structured approach is helpful that includes a detailed assessment of (a) the hippocampus and mesial temporal lobe structures, (b) the ventricular outline, and (c) the gyral and the sulcal anatomy.

- Particular emphasis should be paid upon the T2/ FLAIR signal within the cortex and hippocampus, its similarity to other regions of neo- and archicortex, the internal architecture of the hippocampus, the indentations of the pes hippocampi, the fornix and mammillary bodies, and the grey-white matter interface of the neocortex (blurring, gray matter thinning or thickening).

- The malformations of cortical development can be differentiated into disorders of neuronal proliferation, migration and cortical organization.

\section{References}

1. Duncan JS. Imaging in the surgical treatment of epilepsy. Nat Rev Neurol. 2010;6:537-50.

2. von Oertzen J, Urbach H, Jungbluth S, et al. Standard magnetic resonance imaging is inadequate for patients with refractory focal epilepsy. J Neurol Neurosurg Psychiatry. 2002;73:643-7. 
3. Téllez-Zentenoa JF, Hernández Ronquilloa L, Moien-Afshari F, et al. Surgical outcomes in lesional and non-lesional epilepsy: a systematic review and meta-analysis. Epilepsy Res. 2010;89:310-8.

4. Wellmer J, Quesada CM, Rothe L, et al. Proposal for a magnetic resonance imaging protocol for the detection of epileptogenic lesions at early outpatient stages. Epilepsia. 2013;54:1977-87.

5. Strandberg M, Larsson EM, Backman S, et al. Pre-surgical epilepsy evaluation using 3T MRI. Do surface coils provide additional information? Epileptic Disord. 2008;10:83-92.

6. Winstona GP, Micallef C, Brian E, Kendell BE, et al. The value of repeat neuroimaging for epilepsy at a tertiary referral centre: 16 years of experience. Epilepsy Res. 2013;105:349-55.

7. Balchandani P, Naidich TP. Ultra-high-field MR neuroimaging. Am J Neuroradiol. 2015;36:1204-15.

8. De Ciantis A, Barkovich AJ, Cosottini M, et al. Ultra-high-field MR imaging in polymicrogyria and epilepsy. Am J Neuroradiol. 2015;36:309-16.

9. Breyer T, Wanke I, Maderwald S, et al. Imaging of patients with hippocampal sclerosis at 7 Tesla: initial results. Acad Radiol. 2010;17:421-6.

10. Neel Madan N, Grant PE. New directions in clinical imaging of cortical dysplasias. Epilepsia. 2009;50:9-18.

11. Bauer PR, Reitsma JB, Bernard M, Houweling BM, et al. Can fMRI safely replace the Wada test for preoperative assessment of language lateralisation? A meta-analysis and systematic review. J Neurol Neurosurg Psychiatry. 2014;85:581-8.

12. Piper RJ, Yoong MM, Kandasamy J, et al. Application of diffusion tensor imaging and tractography of the optic radiation in anterior temporal lobe resection for epilepsy: a systematic review. Clin Neurol Neurosurg. 2014;124:59-65.

13. Desai A, Bekelis K, Thadani VM, et al. Interictal PET and ictal subtraction SPECT: sensitivity in the detection of seizure foci in patients with medically intractable epilepsy. Epilepsia. 2013;54:341-50.

14. Barkovich AJ, Guerrini R, Kuzniecky RI, et al. A developmental and genetic classification for malformations of cortical development: update. Brain. 2012;135:1348-69.

15. Howe KL, Dimitri D, Heyn C, et al. Histologically confirmed hippocampal structural features revealed by $3 \mathrm{~T}$ MR imaging: potential to increase diagnostic specificity of mesial temporal sclerosis. Am J Neuroradiol. 2010;31:1682-9.

16. Kimura Y, Shioya A, Saito Y, et al. Radiologic and pathologic features of the transmantle sign in focal cortical dysplasia: the T1 signal is useful for differentiating subtypes. Am J Neuroradiol. 2019;40:1060-6.

17. Sato N, Ota M, Yagishita A, Miki Y, et al. Aberrant midsagittal fiber tracts in patients with hemimegalencephaly. Am J Neuroradiol. 2008;29:823-7.

\section{Suggested Readings}

Gaitanis JN, Donahue J. Focal cortical dysplasia. Pediatr Neurol. 2013;49:79-87.

Guerrini R, Duchowny M, Jayakar P, et al. Diagnostic methods and treatment options for focal cortical dysplasia. Epilepsia. 2015;56:1669-86.

Pohlmann-Eden B, Crocker CE, Matthias H, Schmidt MH. A conceptual framework for the use of neuroimaging to study and predict pharmacoresistance in epilepsy. Epilepsia. 2013;54:75-9.

Ryvlin P, Cross JH, Rheims S. Epilepsy surgery in children and adults. Lancet Neurol. 2014;13:1114-26.

Vattoth S, Manzil FFP, Singhal A, et al. State of the art epilepsy imaging an update. Clin Nucl Med. 2014;39:511-26.

Open Access This chapter is licensed under the terms of the Creative Commons Attribution 4.0 International License (http://creativecommons. org/licenses/by/4.0/), which permits use, sharing, adaptation, distribution and reproduction in any medium or format, as long as you give appropriate credit to the original author(s) and the source, provide a link to the Creative Commons license and indicate if changes were made.

The images or other third party material in this chapter are included in the chapter's Creative Commons license, unless indicated otherwise in a credit line to the material. If material is not included in the chapter's Creative Commons license and your intended use is not permitted by statutory regulation or exceeds the permitted use, you will need to obtain permission directly from the copyright holder. 